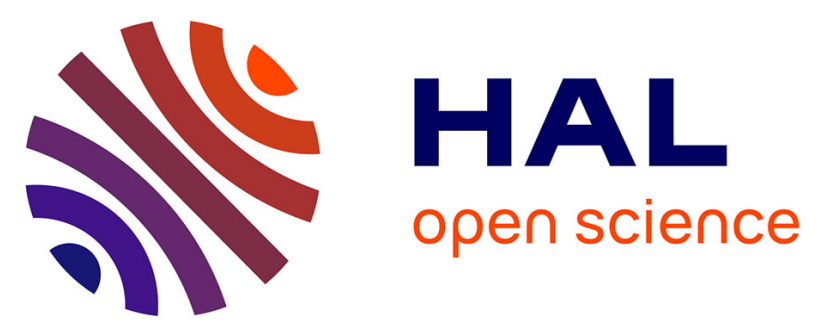

\title{
RACOON++: A Semi-Automatic Framework for the Selfishness-Aware Design of Cooperative Systems
}

Guido Lena Cota, Sonia Ben Mokhtar, Gabriele Gianini, Ernesto Damiani, Julia L. Lawall, Gilles Muller, Lionel Brunie

\section{- To cite this version:}

Guido Lena Cota, Sonia Ben Mokhtar, Gabriele Gianini, Ernesto Damiani, Julia L. Lawall, et al.. RACOON++: A Semi-Automatic Framework for the Selfishness-Aware Design of Cooperative Systems. IEEE Transactions on Dependable and Secure Computing, 2019, 16 (4), pp.635-650. 10.1109/TDSC.2017.2706286 . hal-02196805

\section{HAL Id: hal-02196805 https://hal.science/hal-02196805}

Submitted on 29 Jul 2019

HAL is a multi-disciplinary open access archive for the deposit and dissemination of scientific research documents, whether they are published or not. The documents may come from teaching and research institutions in France or abroad, or from public or private research centers.
L'archive ouverte pluridisciplinaire HAL, est destinée au dépôt et à la diffusion de documents scientifiques de niveau recherche, publiés ou non, émanant des établissements d'enseignement et de recherche français ou étrangers, des laboratoires publics ou privés. 


\title{
RACOON++: A Semi-Automatic Framework for the Selfishness-Aware Design of Cooperative Systems
}

\author{
Guido Lena Cota, Sonia Ben Mokhtar, Gabriele Gianini, Ernesto Damiani, \\ Julia Lawall, Gilles Muller, and Lionel Brunie
}

\begin{abstract}
A challenge in designing cooperative distributed systems is to develop feasible and cost-effective mechanisms to foster cooperation among selfish nodes, i.e., nodes that strategically deviate from the intended specification to increase their individual utility. Finding a satisfactory solution to this challenge may be complicated by the intrinsic characteristics of each system, as well as by the particular objectives set by the system designer. Our previous work addressed this challenge by proposing RACOON, a general and semi-automatic framework for designing selfishness-resilient cooperative systems. RACOON relies on classical game theory and a custom built simulator to predict the impact of a fixed set of selfish behaviours on the designer's objectives. In this paper, we present RACOON++, which extends the previous framework with a declarative model for defining the utility function and the static behaviour of selfish nodes, along with a new model for reasoning on the dynamic interactions of nodes, based on evolutionary game theory. We illustrate the benefits of using RACOON++ by designing three cooperative systems: a peer-to-peer live streaming system, a load balancing protocol, and an anonymous communication system. Extensive experimental results using the state-of-the-art PeerSim simulator verify that the systems designed using RACOON++ achieve both selfishness-resilience and high performance.
\end{abstract}

\section{INTRODUCTION}

T $\mathrm{N}$ recent years, the importance of cooperative systems 1 such as peer-to-peer (P2P) networks and collaborative computing has rapidly grown, driven by multiple factors. First, the ever-increasing demand for video content [1] poses serious challenges to the operational and economic sustainability of traditional content delivery networks [2], paving the way for more scalable, robust and cost-effective P2P-assisted solutions [3]. Second, cooperative systems are the key enablers of new and emerging technologies, including the blockchain ecosystem [4] and the Internet of Things [5]. Finally, the decentralised nature of cooperative systems can address the increasing privacy concerns of their users [6], by avoiding control and potential misuse of sensitive data by a centralised server.

Crucial to the success of cooperative systems is that nodes are willing to collaborate with each other by sharing part of their resources-e.g., network bandwidth, storage

- G. Lena Cota and G. Gianini are with the Università degli Studi di Milano, Milano 20122, Italy. E-mail: \{guido.lena, gabriele.gianini\}@unimi.it.

- S. Ben Mokhtar and L. Brunie are with LIRIS-CNRS-INSA Lyon, Villeurbanne 69622, France.

E-mail: sonia.ben-mokhtar@liris.cnrs.fr,Lionel.Brunie@insa-lyon.fr.

- J. Lawall and G. Muller are with Sorbonne Universités, Inria, CNRS, UPMC, LIP6, Paris 75005, France.

E-mail: \{julia.lawall, gilles.muller\}@lip6.fr.

- E. Damiani is with the Università degli Studi di Milano, Milano 20122, Italy, and the EBTIC/Khalifa University, Abu Dhabi 127788, UAE.

E-mail:ernesto.damiani@unimi.it.

Manuscript received 22 Aug. 2016; revised 27 Mar. 2017; accepted 8 May 2017. Date of publication 0 .0000; date of current version 0.0000. (Corresponding author: Guido Lena Cota.)

For information on obtaining reprints of this article, please send e-mail to: reprints@ieee.org, and reference the Digital Object Identifier below.

Digital Object Identifier no. 10.1109/TDSC.2017.2706286 space, CPU time. However, in practice [7], [8], [9], real sys- 35 tems often suffer from selfish nodes that strategically with- 36 draw from cooperation to satisfy their individual interests 37 at the expense of the system reliability and efficiency. In 38 fact, several studies have shown that selfishness in coopera- 39 tive systems results in substantial degradation of perfor- 40 mance, unpredictable or limited availability of resources, 41 and may even lead to a complete disruption of the system 42 functionalities [13], [23], [25]. For example, Guerraoui 43 et al. [14] observed experimentally that if 25 percent of 44 nodes in a P2P live streaming system download a given 45 video file without sharing it with other nodes, then half of 46 the remaining nodes are not able to view a clear stream. $\quad 47$

Different solutions have been proposed to deal with self- 48 ishness in cooperative systems [22], [23], [24], [25], [26], [27], 49 [28]. Most of these solutions rely on Game Theory (GT), a 50 theoretical framework to model and study selfish behav- 51 iours [31]. The typical approach to design selfishness- 52 resilient systems using GT requires first creating an analyti- 53 cal model of the system (the game) and then proving mathe- 54 matically that the cooperative behaviour is the best strategy 55 for selfish nodes (a Nash Equilibrium), with respect to a 56 known utility function. However, carrying out this process 57 is complex, error-prone, and time-consuming [30].

Detecting and punishing selfish behaviours at runtime is 59 an alternative, more practical approach. Diarra et al. [13] 60 showed that making nodes accountable for their actions can 61 be a strong incentive for selfish nodes to cooperate. In an 62 accountable system, each node maintains a secure log to 63 record its interactions with other nodes. Also, each node is 64 associated with a set of witness nodes that periodically check 65 whether the log entries correspond to a correct execution of 66 
67 the system. If any deviation is detected, then the witnesses 68 build a proof of misbehaviour that can be verified by any 69 correct node, and punishment is inflicted on the misbehav70 ing one. Although accountability mechanisms have been 71 successfully applied to cooperative systems [12], [13], [14], 72 the additional work required at each node (e.g., crypto73 graphic operations, log auditing) can significantly increase 74 computation, bandwidth, and storage requirements. More75 over, the fine tuning of these mechanisms for building a 76 selfishness-resilient and cost-effective cooperative system 77 could be a challenging task [19].

Configuring accountability mechanisms requires that a system designer select values for a number of parameters (e.g., number of witnesses, audit frequency) that directly affect the system performance (e.g., bandwidth usage, delay). In the literature [12], [13], [14], no indication is provided for the setting of these parameters, leaving entirely to designers to find a configuration that achieves the desired level of resilience to selfish behaviours while imposing minimal overhead. Finding this critical trade-off involves the systematic evaluation of a large number of experiments, to investigate the impact of the value of each parameter on the system performance. Moreover, such experiments require the ability to create and inject selfish behaviours, which is not supported by state-of-the-art experimental environments, such as Splay [41], NS-3 [39], and PeerSim [10].

To address the design challenges discussed above, our previous work [19] proposed RACOON, a general framework for designing efficient $\mathrm{P} 2 \mathrm{P}$ systems resilient to selfish nodes in a semi-automatic manner. To begin, the designer provides the functional specification of the system (i.e., communication protocols) and a set of performance objectives. RACOON uses this information to mostly automate the following steps: (i) enforce practical mechanisms to foster cooperation (i.e., distributed accountability and reputation mechanisms), (ii) identify possible selfish deviations from the functional specification; (iii) develop a behavioural model of the system participants as a non-cooperative game [31], to predict the strategic choices of selfish nodes; and (iv) tune the accountability and reputation parameters to meet the designer's objectives, using GT-based simulations. Each step is carried out by a distinct module of the framework, which can be replaced or extended with a new definition (e.g., different models for selfishness). RACOON results in a complete design of the system, which includes finely tuned mechanisms to meet selfishness-resilience and performance objectives. This output serves as a reference to developers for the eventual implementation of the system.

In this paper, we describe $R A C O O N++$, which extends the previous version of our framework by addressing a number of limitations and introducing new features. First, we provide the designers with a simple yet expressive specification model to define the utility function and the behaviour of selfish nodes, which in $R A C O O N$ were predefined and fixed for all application scenarios. This model shapes the utility function of a node by assigning costs and benefits to specific actions of the communication protocols, and parametrises some aspects of selfish behaviours (who deviates, from which action, with what type of deviation). Second, we model the behaviour of selfish nodes using Evolutionary Game Theory (EGT) [32] instead of the classical GT used in RACOON. Using EGT, we can relax the assumption of perfect rationality of the nodes, 128 and consider them as active learners who adjust their strategy 129 over time in response to repeated observations of their own 130 and others' utilities. Such learning and adaptation processes 131 better reflect with the computational and decisional capabili- 132 ties of real nodes [28], [29]. Furthermore, as noted by Palomar 133 et al. [27], an evolutionary approach is more appropriate for 134 modelling the dynamic behaviour of cooperative systems. 135 Third, we integrate the RACOON++ functionalities with the 136 P2P simulator PeerSim [10]. To the best of our knowledge, the 137 simulator we developed in RACOON was the first tool able to 138 dynamically simulate selfish strategic behaviours. However, 139 like all custom built simulators, it had neither the maturity 140 nor the acceptance of state-of-the-art tools like PeerSim [39]. $\quad 141$

In summary, we present the following contributions: 142

- Selfishness-aware design of cooperative systems. We 143 define simple declarative models for specifying 144 cooperative protocols as well as for describing 145 nodes' selfishness.

- Automatic (evolutionary) game-theoretic reasoning. We 147 define the system under design as an evolutionary 148 game, in order to describe how successful behav- 149 iours spread in a population of selfish individuals. 150 We also provide an automatic methodology to gen- 151 erate the game using the information contained in 152 the declarative models. Finally, we extend the Peer- 153 Sim simulator with the ability to conduct EGT analy- 154 sis to simulate selfish behaviours. 155 Objective-oriented configuration. We propose an auto- 156 matic configuration method for an accountability and 157 reputation mechanism in a cooperative system, 158 which can meet the resilience and performance objec- 159 tives set by a system designer in a reasonable time for 160 a design-time activity (18 minutes on average). 161

- Generality, simplicity and performance. We assess the 162 design effort and effectiveness of using RACOON++ 163 on three use cases: a P2P live streaming system [14], a 164 P2P load balancing protocol [10], and an anonymous 165 communication system based on Onion Routing [15]. 166

The rest of the paper is organised as follows. Section 2167 reviews the related work. Section 3 presents an overview of 168 RACOON++, followed by a detailed description of its two 169 phases: the design phase (Section 4) and the tuning phase 170 (Section 5). Section 6 summarises the operation of the frame- 171 work from the designer's point of view. Section 7 presents a 172 performance evaluation of $R A C O O N++$. Finally, the paper 173 concludes in Section 8.

\section{Related Work}

Game Theory is a mathematical framework to model and 176 predict the interactions among selfish and strategic individu- 177 als [31]. Much work on GT as a tool for system designers has 178 been carried out in the context of content dissemination [24], 179 [25], [26], [27], wireless and mobile networking [20], [21], 180 cryptography, anonymity and privacy mechanisms [16], 181 [17], [18], [23]. The objective of these works is to make coop- 182 eration the best choice for all nodes, i.e., a Nash Equilibrium. 183 Most of the GT solutions are not readily applicable to cooper- 184 ative systems [30], mainly due to simplifying assumptions to 185 make the model tractable, e.g., assuming that nodes have 186 
DESIGN

TUNING

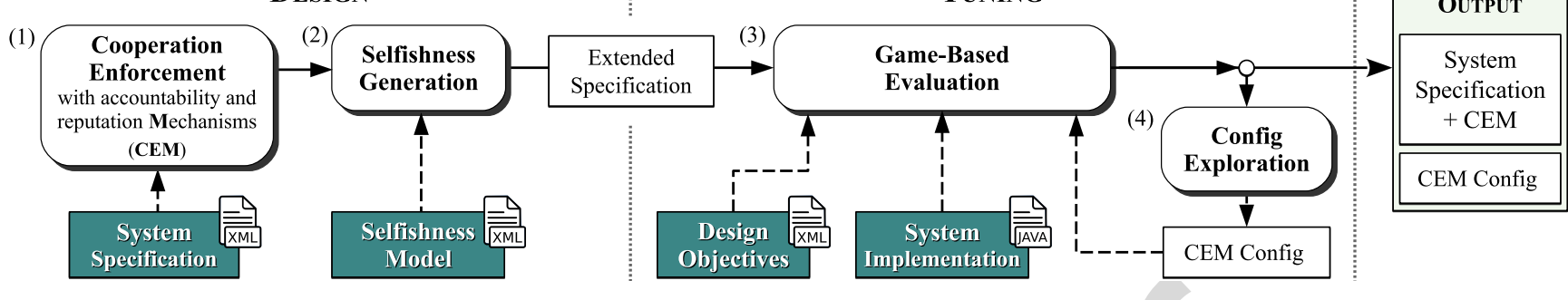

Fig. 1. Overview of the RACOON++ framework.

perfect rationality [18], [19], [20] or are risk averse [23], [24], [25]. Evolutionary Game Theory (EGT) relaxes these assumptions by considering nodes with limited rationality that adapt their behaviours dynamically, by learning from experience [32]. However, most applications of EGT to system design are only suitable for toy systems [27], [28], [29], because of the difficulty of modelling a complex system in a formal way. RACOON++ provides means for transforming models familiar to system designers (state machines) into games, thus making the power of EGT reasoning accessible to non-game theory experts.

Another common limitation of GT models is that they are tailored to a specific system problem and are difficult to adapt to a changing environment. A notable example is the BAR Model for designing systems robust to selfish and Byzantine participants [22]. Besides the difficulties in the manual design of a BAR-tolerant system [22], [23], [24], [25], the resulting solution suffers from poor flexibility and maintainability. Every change to the system parameters requires a full revision of the design, hindering the reuse of a successful solution in other systems. On the contrary, the general approach of $\mathrm{RACOON++}$, as well as its applicationindependent mechanisms to enforce cooperation, are reusable by construction. Furthermore, RACOON++ supports a semi-automatic design flow that greatly facilitates the refinement of system requirements and specification.

Yumerefendi and Chase [34] advocate accountability as a viable solution for dealing with non-cooperative behaviours. Distributed accountability mechanisms [12], [13], [14], notably FullReview [13], have been proven effective in systems populated by selfish nodes, making them an ideal and general component for supporting cooperation in RACOON++. However, enforcing accountability incurs a substantial cost on the system, mainly due to the high message overhead and the intensive use of cryptography. This poses a significant configuration problem, requiring designers to carefully look for a trade-off between performance and selfishnessresilience. Since no guidelines are given in the studies cited above, tuning the accountability mechanisms is manual and time-consuming. In contrast, $R A C O O N++$ mostly automates this task.

Accountability systems usually address selfishness by isolating or evicting selfish nodes [13], [14]. A complementary approach is to introduce incentives to make cooperation more profitable for selfish nodes. The vast body of literature on incentives for cooperative systems can broadly be divided into trust-based and trade-based incentive schemes. A trust-based scheme associates each node with a level of trust, which can serve as a guide for distributing incentives. For example, nodes with a high trust level can benefit from a higher quality of service. Reputation is the 237 principal mechanism to evaluate and maintain trust in 238 dynamic large-scale environments like cooperative sys- 239 tems [37]. Reputation mechanisms offer high flexibility and 240 scalability, and can be implemented in a fully decentralised 241 manner. Because of these features, RACOON++ uses a dis- 242 tributed reputation mechanism to foster cooperation, which 243 complements the trust-enabling approach of its accountabil- 244 ity system. Specifically, the reputation of nodes is updated 245 based on verifiable evidence and linked to a unique and 246 permanent identity, thereby inhibiting the dissemination of 247 false information (e.g., bad mouthing, unfair praise) [38]. ${ }^{1} \quad 248$

In trade-based incentive schemes, nodes pay for obtain- 249 ing services or resources (as consumers) and get paid for 250 sharing (as providers). In schemes such as barter and tit-for- 251 tat [11], [20], [25], the trade is direct and symmetric: each 252 unit of resource is reciprocated with a unit of resource. 253 Although very robust and easy to implement, these schemes 254 require that trading nodes need something from each other 255 (a condition known as double coincidence of wants) and 256 that they establish long duration relationships to ensure 257 adequate opportunities for reciprocation. These require- 258 ments can be too restrictive or inefficient in some coopera- 259 tive systems, such as opportunistic networks [20] and real- 260 time constrained applications [24]. To overcome this limita- 261 tion, credit-based mechanisms [35], [36] use virtual currency 262 as the commodity for trading resources and allowing its 263 later expenditure. On the downside, these approaches intro- 264 duce economic issues in the system (e.g., price negotiation, 265 inflation, deflation) [36], and may require a trusted author- 266 ity (bank) to issue and certify the currency [35]. By contrast, 267 RACOON++ uses fully distributed mechanisms that are not 268 affected by economic factors.

Several frameworks and domain-specific languages have 270 been proposed to ease the task of designing and evaluating 271 dependable distributed systems (e.g., [40], [41]). Although 272 these solutions yield good results in terms of system perfor- 273 mance and designer effort, none of them addresses the specific 274 threat of selfish deviations in cooperative distributed systems. 275

\section{RACOON++: OVERVIEW}

RACOON++ is a design and simulation framework aimed 277 at supporting system designers in building a selfishness- 278 resilient cooperative system that meets desired performance 279 objectives. As depicted in Fig. 1, the operation of RACOON++ 280

1. Although out of the scope of our present work, it is worth noting that strong identities are the prerequisite for preventing other strategic misbehaviours against reputation systems, such as whitewashing and Sybil attacks [34], [38]. 

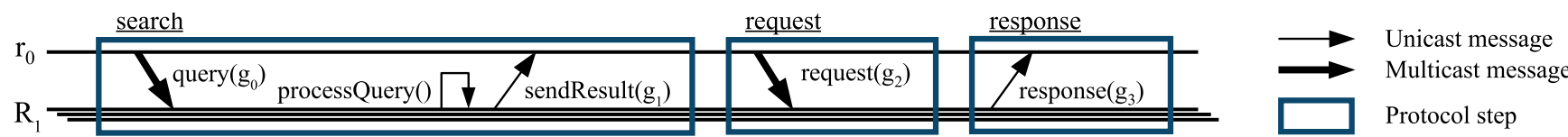

Fig. 2. The S-R-R protocol between nodes $r_{0}$ and $R_{1}$.

consists of two phases: the assisted design of the system and the objective-oriented tuning of its parameters. The dark boxes in Fig. 1 are the input provided by the designer. We give an overview of these phases here, and more details in Sections 4 and 5.

The design phase is initiated by the system designer (hereafter "Designer", for brevity), who provides a statemachine specification of the communication protocols composing the cooperative system. In Step (1) of Fig. 1, $\mathrm{RACOON++}$ integrates the system specification with mechanisms to encourage nodes to cooperate. Specifically, RACOON++ uses two general and configurable Cooperation Enforcement Mechanisms (CEM): an accountability system to audit nodes' behaviour and a reputation system to assign rewards or punishments depending on the audit results. Then, the framework extends the state machine representation of the system by adding new states and transitions that represent selfish behaviours (Step (2)). For a better control over the process, the Designer can describe the preferences and capabilities of selfish nodes using the Selfishness Model. The result is an Extended Specification of the cooperative system, which includes selfish behaviours and cooperation enforcement mechanisms.

The goal of the tuning phase is to find a configuration setting for the CEM that makes the Extended Specification meet a list of Design Objectives set by the Designer. Tuning is an iterative refinement process consisting of a sequence of two steps: game-based evaluation and configuration exploration (Steps (3) and (4) in Fig. 1). The evaluation is done using game theory-driven simulations, carried out automatically by our framework. More precisely, RACOON++ transforms the Extended Specification into a game model, which it uses to simulate the strategic behaviour of selfish nodes given an implementation of the system specification by the Designer. The framework uses the results of the evaluation to traverse the configuration space and evaluate new configuration candidates for the CEM. The output of RACOON++ is a new specification of the cooperative system that includes finely tuned accountability and reputation mechanisms to achieve the selfishness-resilience and performance objectives set by the Designer. This output provides a reference guide for developers to use when implementing the system.

\section{RACOON++ Design Phase}

The design phase helps the Designer in specifying a cooperative system that embeds mechanisms for fostering cooperation as well as in defining a behavioural model of the system participants. The output is a new artefact called the Extended Specification of the system.

In this section, we introduce the inputs of the phase, we describe the accountability and reputation mechanisms used in $R A C O O N++$, and, finally, we present the algorithm used to generate selfish deviations. To support the description of the framework, we use the simple communication protocol Search, Request E Response (S-R-R) shown in Fig. 2. 334 In the S-R-R protocol, a node $r_{0}$ queries other nodes for 335 some desired resources (e.g., files). To this end, $r_{0}$ sends a 336 query message $g_{0}$ to a set of nodes collectively named $R_{1} 337$ (the capital letter denotes a set of nodes). Each node in $R_{1} 338$ processes the query and replies with the list of available 339 resources (message $g_{1}$ ). Upon receiving the list, $r_{0}$ sends a 340 new message $g_{2}$ to $R_{1}$, requesting (a subset of) the resources 341 listed in $g_{1}$. Finally, each node in $R_{1}$ sends the requested 342 data (message $g_{3}$ ).

\subsection{Input of the Design Phase}

The inputs of the design phase are the functional specification 345 of the protocols of the system that should be made resilient 346 to selfish behaviours, and the selfishness model adopted by 347 selfish nodes.

\subsubsection{Functional Specification}

The functional specification describes the correct, cooperative 350 behaviour of nodes by means of communication protocols. 351 Like many other approaches [12], [22], [40], notably the 352 accountability system [13] that we plan to adapt for our 353 framework, each communication protocol is specified using 354 a notation based on deterministic finite state machines, called 355 a Protocol Automaton. A Protocol Automaton PA is a tuple 356 $\langle R, S, T, M, G, C\rangle$, with each component described below. $\quad 357$

Roles $(R)$. The parties involved in the protocol execution. 358 A role determines the responsibilities of a party (a node or a 359 group of nodes) and constrains the actions that the party is 360 allowed to execute in a protocol run. Every PA has at least 361 two types of roles: the requester of a resource or service, 362 and the provider. Other types are also possible (e.g., 363 brokers, auditors, recommenders). For example, the S-R-R 364 protocol has two roles: the requester $r_{0}$ and the set of poten- 365 tial providers $R_{1}$. Formally, a role $r \in R$ is a tuple 366 $\langle r I d$, cardinality, rType $\rangle$, where cardinality denotes the 367 number of nodes represented by $r$, and rType is either 368 requester, provider, or other.

States $(S)$. The set of states that the system goes through 370 when implementing the communication protocol. A state 371 $s \in S$ is a tuple $\langle s I d$, roleId,sType $\rangle$, where roleId identifies 372 the role $r \in R$ that triggers a change of state or terminates 373 the protocol execution, and sType is either initial, final, or 374 intermediate.

Transitions (T). A transition represents a protocol step, 376 i.e., the set of method calls that determine the next protocol 377 state. The PA supports three types of transition: abstract, 378 communication, and computation. An abstract transition 379 groups many method calls into a single "black box" transi- 380 tion, which may simplify the protocol representation by 381 hiding some implementation details. The remaining transi- 382 tion types allow to define the (communication or computa- 383 tion) method that triggers the transition. Formally, a 384 transition $t \in T$ is a tuple $\langle t I d$, state $1 I d$, state $2 I d$, methodId $\rangle, 385$ 


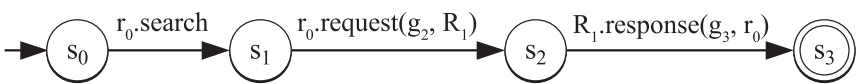

Fig. 3. The protocol automaton of the S-R-R protocol.

where state $1 I d$ and state $2 I d$ identify the source and target states in $S$, and methodId identifies the method executed in $t$ (null, for abstract transitions). In the S-R-R protocol, the transitions are search (abstract), request and response (communication).

Methods (M). The set of actions that can trigger a protocol transition. A communication method represents the delivery of a message from one role to another, whereas a computation method performs local computations. A method $m \in M$ is a tuple $\langle m I d$, message $I d\rangle$, where messageId is defined only for communication methods, and null otherwise. For instance, request is the communication method of the S-R-R protocol that sends a message $g_{2}$ to $R_{1}$. Note that the methods called during an abstract transition (e.g., search) are not in $M$.

Messages (G): A message $g \in G$ sent by a communication method is a tuple $\langle g I d$, senderId, receiverId, contentId $\rangle$, where senderId and receiver Id identify the interacting roles in $R$, and contentId refers to the content $c \in C$ carried by $g$.

Contents (C): A content $c \in C$ is a collection of data units (e.g., integers, binary files), formalised as the tuple $\langle c I d, c$ Type, $c$ Length $\rangle$, which defines the data type $c$ Type ${ }^{2}$ and the number $c$ Length of data units comprising the content.

Fig. 3 shows the state diagram of the S-R-R protocol. The labels on each transition indicate the role and the method that trigger the transition, along with the message sent (if any). For example, the label between states $s_{1}$ and $s_{2}$ indicates that role $r_{0}$ invokes the communication method request to send the message $g_{2}$ to $R_{1}$. The label of an abstract transition indicates the role that executes the first method encapsulated in it.

\subsubsection{Selfishness Model}

The selfishness model carries the information about the economic drivers of a party, by specifying the utility that a node obtains in participating in the system. Also, it defines the possible deviations from the functional specification. Formally, a selfishness model is a tuple $\langle V, D\rangle$, detailed below.

Valuations $(V)$. The set of contributions to the overall utility of a certain behaviour. The utility that a node receives by participating in the system is given by the benefit obtained by consuming resources and the cost of sharing resources. A valuation $v \in V$ specifies this information at the granularity of transitions and messages of a Protocol Automaton. Formally, $v$ is a tuple $\langle v I d, v$ Scope, roleId, benefit, cost $\rangle$, where vScope is the identifier of the PA element (transition or message) that brings some bene fit and cost (numeric values) to the role in $R$ identified by roleId.

If the $v$ Scope of a valuation $v_{t}$ refers to a transition $t \in T$, then $v_{t}$ defines the utility that the role with identifier $v_{t}$.roleId obtains by executing $t$. We denote by $v\left(v_{t}\right)$ the function to evaluate the contribution of $v_{t}$ to the overall utility, and we define it as: $v\left(v_{t}\right)=v_{t}$. benefit $-v_{t}$.cost. As an example, consider the search transition of the S-R-R protocol. It is

2. Defined by the XML Schema type system. reasonable to expect that role $r_{0}$ receives more benefit than 439 cost from the transition, because the node will eventually 440 receive useful information. This consideration can be 441 expressed by the valuation $\left\langle v_{0}\right.$, search, $\left.r_{0}, 10,1\right\rangle$, which 442 results in a contribution to the utility of $v\left(v_{0}\right)=9$. Note that 443 another system designer may value the same transition dif- 444 ferently, according to her expertise and knowledge of the 445 system.

Conversely, if the $v$ Scope of a valuation $v_{g}$ refers to a mes- 447 sage $g \in G$, then $v_{g}$ defines the utility obtained by the role 448 identified in $v_{g}$ when $g$ is sent or received. The contribution 449 of $v_{g}$ to the overall utility accounts for the cardinality of the 450 receiver role of the message as well as the number of data 451 units comprising the delivered content. This is based on the 452 observation that the costs and benefits of a message are typi- 453 cally proportional to the number of data units transmitted 454 or received (e.g., the communication costs of a message 455 depends on its length and number of recipients). Consider, 456 for instance, the request transition of the S-R-R protocol, 457 which involves the transmission of a message $g_{2}$ to role $R_{1}$. 458 Let $c_{2}$ be the content transmitted by $g_{2}$, and let 459 $\left\langle v_{1}, g_{2}, r_{0}, 5,1\right\rangle$ be the valuation associated with $g_{2}$. In this 460 case, the contribution that $v_{1}$ makes to the utility of the node 461 playing the role of $r_{0}$ is given by: $v\left(v_{1}\right)=(5-1) \cdot 462$ $c_{2}$.cLength $\cdot R_{1}$.cardinality. Note that it is also possible to 463 define a valuation associated to $g_{2}$ that specifies benefits 464 and costs of the receiver $R_{1}$ of the message; for instance, 465 $v_{2}=\left\langle v_{2}, g_{2}, R_{1}, 1,0\right\rangle$. 466

Selfish Deviations (D). The set of deviations from the cor- 467 rect execution of the system, made by selfish nodes to 468 increase their utility. In the context of a cooperative system, 469 a selfish node can increase its utility by reducing the cost of 470 sharing resources. Concretely, a deviation can reduce the 471 bandwidth consumption by sending fewer and shorter mes- 472 sages [7], [8], [14], [23], or interrupt resource contribution by 473 refusing to execute some methods [9], [13], [23]. Based on 474 the study of these and other examples from the literature, 475 we have selected the three generic types of selfish deviation 476 supported by $R A C O O N++$, namely: (1) timeout deviation: a 477 node does not implement the prescribed transition within 478 the time limit; (2) subset deviation: a node sends a subset of 479 the correct message content; and (3) multicast deviation: a 480 node sends a message to a random subset of the legitimate 481 recipients. Some other types of selfishness, notably collusion 482 and provision of false or misleading information, have been 483 investigated in our recent work [42].

Formally, a selfish deviation $d \in D$ from a transition 485

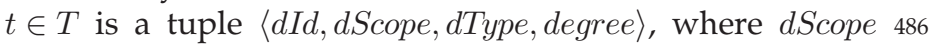
identifies $t$, dType indicates whether $d$ is a timeout, subset or 487 multicast deviation, and degree $\in[0,1]$ specifies the inten- 488 sity of the deviation. Note that the timeout deviation can 489 affect all types of transitions, whereas the subset and multi- 490 cast deviations affect only communication transitions, as 491 they interfere with the delivery of a message. For instance, 492 $\left\langle d_{0}\right.$, response, timeout, 1$\rangle$ describes selfish nodes that never 493 reply to a request. Note that timeout deviations only occur to 494 the maximum degree. As another example, suppose the 495 Designer wants to account for selfish nodes that only send 496 half of the content in any message exchange of the S-R-R 497 protocol (e.g., half of the requested resources). The selfish 498 deviation $\left\langle d_{1}, *\right.$, subset, 0.5$\rangle$ represents this behaviour, 499 


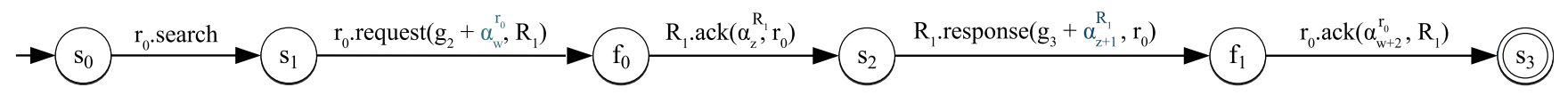

Fig. 4. The integration between the commitment protocol of $R$-acc with the S-R-R protocol shown in Fig. 3.

where the wildcard value "**" indicates that all communication transitions in the PA are subject to $d_{1}$.

\subsection{Cooperation Enforcement}

The first automatic step of the design phase of RACOON++ is the integration of the Cooperation Enforcement Mechanisms into the functional specification provided by the Designer. The CEM includes accountability and reputation protocols to make cooperation the most profitable behaviour for all nodes. In practice, the quality of service received by nodes depends on their reputation values, which are updated based on accountability audits. To this end, the CEM imposes an allocation regime such that the probability of a node receiving a service or a resource in the future is proportional to its current reputation. If the reputation of a node hits the lower bound, no other node will accept its requests, thus preventing the node from receiving any benefit from the system. The advantage of such a flexible incentive scheme is twofold. First, and with respect to rigid punishment strategies such as direct eviction [13], it alleviates the impact of false-positive detection of selfish nodes [19]. Second, all nodes are evaluated based on the quality of their contribution (cooperative or selfish) rather than on the quantity of the shared resources, so as not to penalise nodes suffering from persistent resource shortage (e.g., battery-powered devices).

The CEM is a key component for the Designer, as it provides general and off-the-shelf mechanisms for fostering cooperation in a wide range of settings, without the need to devise ad-hoc solutions for the particular system at hand.

Hereafter, we discuss the CEM used in RACOON++.

\subsubsection{Accountability Mechanism}

$\mathrm{RACOON++}$ uses accountability techniques for detecting misbehaviours and assigning nodes non-repudiable responsibility for their actions. Specifically, we propose the $R$-acc mechanism, based on the FullReview [13] protocols and architecture. $R$-acc also shares some assumptions with FullReview about nodes' behaviours (i.e., no collusion) and the system (i.e., a Public Key Infrastructure is available to create trusted identities by means of digital signatures), whereas it differs on other assumptions (e.g., nodes are not risk averse).

$R A C O O N++$ can automatically integrate $R$-acc into the functional specification provided by the Designer. To begin, $R$-acc requires each node to maintain a tamper-evident record of all its observable actions (i.e., message exchanges). Further, each node is assigned to a set of other nodes, called its witness set. A witness is in charge of auditing the log of its monitored nodes, generating provable evidence of their 546 behaviours and assigning punishments or rewards accord- 547 ingly. Such operations are defined by the protocols 548 described below.

Commitment Protocol. Ensures that the sender and the 550 receiver of a message have provable evidence that the other 551 party has logged the exchange. Fig. 4 shows the integration 552 between the PA of the S-R-R protocol and the commitment 553 protocol. Consider for example the node with role $r_{0}$ in state 554 $s_{1}$. Before sending the request message $g_{2}$ to $R_{1}, r_{0}$ records 555 the action in its local log, creating a new entry $e_{w}$. Then, $r_{0} 556$ generates a signed statement $\alpha_{w}^{r_{0}}$, called an authenticator, 557 indicating that it has logged $e_{w}$. Next, $r_{0}$ sends $\alpha_{w}^{r_{0}}$ to $R_{1} 558$ along with the message. Upon receiving the message (state 559 $f_{0}$ in Fig. 4), each node in $R_{1} \operatorname{logs}$ this event in a new $\log 560$ entry $e_{z}$, and generates the corresponding authenticator $\alpha_{z}^{R_{1}} .{ }_{561}$ Finally, $R_{1}$ sends this authenticator to $r_{0}$ to acknowledge the 562 reception of $g_{2}$.

Audit Protocol. A proactive and periodic inspection of a 564 node's behaviour, based on the examination of its log. In 565 contrast with FullReview, $R$-acc introduces the probability of 566 audit parameter, which allows more control over the num- 567 ber of audits, instead of auditing at every audit period. 568 Fig. 5 shows the PA of the audit protocol between a moni- 569 tored node $r_{m}$ and one of its witnesses $r_{w}$. Upon receiving 570 the audit request $g_{a 0}$, the witness requests and obtains a por- 571 tion of the $\log$ of $r_{m}$ (messages $g_{a 1}$ and $g_{a 2}$ ). Then, $r_{w}$ verifies 572 if $r_{m}^{\prime}$ 's log conforms to the correct behaviour making up the 573 functional specification of the system (transition audit in 574 Fig. 5). The witness sends the audit result back to the moni- 575 tored node (message $g_{a 3}$ ). Finally, $r_{m}$ checks the correctness 576 of its audit by forwarding the collected results to the witness 577 set of each of its witnesses (indicated as $w\left(r_{w}\right)$ in the figure). 578 If the witness does not receive the requested log from $r_{m} 579$ (state $f_{7}$ in Fig. 5), then it will address the issue by using the 580 challenge/response protocol.

Consistency Protocol. Ensures that each node maintains a 582 single and consistent linear log [13].

Challenge/Response Protocols. Deal with nodes that do not 584 respond to messages as provided in PA or in $R$-acc, allowing 585 certain tolerance for correct nodes that are slow or suffering 586 from network problems (e.g., message loss). Specifically, if a 587 node $i$ has been waiting too long for a given message from 588 another node $j, i$ indicates the suspect state for $j$, and creates 589 a challenge for it. In FullReview, nodes communicate only 590 with non-suspected nodes. $R$-acc adopts a more tolerant 591 approach: while in the suspect state, the probability of $j$ to 592 communicate with $i$ is locally decreased by a fixed amount, 593 until $j$ responds to the challenge and gets trusted again.

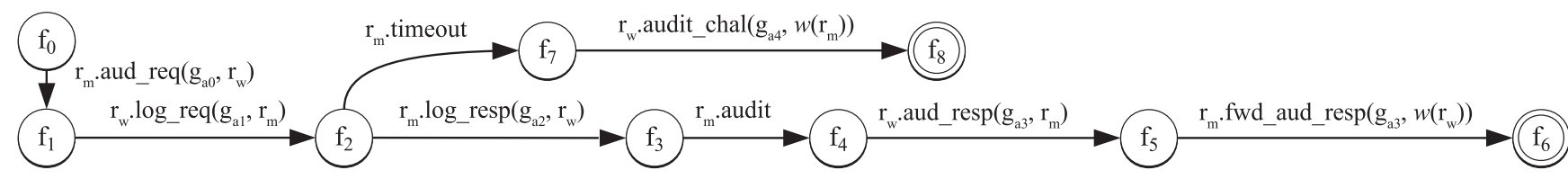

Fig. 5. The Protocol Automaton of the R-Acc audit protocol. 
$R$-acc does not include the evidence transfer protocol used in FullReview [13]. The same goal of ensuring that faulty nodes are eventually exposed by all correct nodes in the system is accomplished by the reputation mechanism described next.

The commitment protocol is the only $R$-acc protocol that modifies the functional specification of the system. The remaining protocols run in separate threads, scheduled to execute periodically. RACOON++ includes a PA specification for each protocol. The Designer can refer to these specifications when writing the selfishness model, to define valuations and deviations also for $R-a c c$, and test whether accountability still holds when this mechanism is enforced by selfish nodes.

\subsubsection{Reputation Mechanism}

The reputation of a node is the summary of its history of behaviours, which is used to assist nodes in choosing a cooperative partner with which to interact. Cooperation leads to a good reputation, whereas selfish behaviours lead to a bad reputation. To provide this information, the CEM includes a distributed reputation mechanism (R-rep) to form, aggregate, and disseminate reputation values.

In order to reduce design complexity and to reuse available knowledge, $R$-rep shares some features with the $R$-acc accountability mechanism described above. First, a witness node plays the role of recommender in $R$-rep, as it can form an opinion of a monitored node based on the audit result. This solution keeps the computational overhead of the CEM under control, as it avoids performing the same operation twice (that is, the evaluation of a certain behaviour). Furthermore, basing feedback on provable evidence offers an effective defence against false feedback (e.g., bad mouthing, false praising) [38]. Second, $R$-rep relies on $R$-acc for storing the reputation data in a reliable manner. More precisely, nodes store their own reputation locally. To prevent manipulations, only witnesses - in their role of recommenders-can update the reputation value. Also, the update must be recorded in the $R$-acc secure log, so that any tampering can be detected.

In $R$-rep, the reputation $\rho$ of a node is an integer value between 0 and an upper limit $\rho_{\max }$. The value of $\rho$ is estimated after every audit, and can be calculated as

$$
\rho=\left\{\begin{array}{ll}
\max \left\{\rho_{\text {old }}-f_{p}\left(\rho_{\text {old }}, d_{p}, d_{d}\right), 0\right\}, & \text { if positive audit } \\
\min \left\{\rho_{\text {old }}+f_{r}\left(\rho_{\text {old }}, d_{r}\right), \rho_{\text {max }}\right\}, & \text { if negative audit }
\end{array},\right.
$$

where $\rho_{\text {old }}$ is the old reputation value, $f_{p}$ and $f_{d}$ are the update functions in case of punishment or rewards, $d_{p}$ and $d_{r}$ are two $R$-rep parameters that control the degree of punishment and of reward, and $d_{d}$ is the degree of the deviations detected by the audit. A punishment comes in the form of a reputation decrease. The decrease value is proportional to $d_{p}$ and to the degree $d_{d}$ of the detected deviation, and indirectly proportional to the old reputation value $\rho_{\text {old }}$, in such a way as to punish with greater severity nodes that already have a bad reputation, in order to inhibit recidivism. In the case of a negative audit, function $f_{r}$ rewards the cooperative node by assigning a reputation increase, which is proportional to the degree of reward $d_{r}$ and to the old reputation value.

Consider for example the following setting of R-rep: $\rho_{\max }$ is 10 , the $d_{r}$ is 0.2 , and $d_{p}$ is 2 . Also, let a currently cooperative node have the reputation value 5. After being 653 audited, the node's reputation value will be $\rho=\min \{5+654$ $(5 \cdot 0.2), 10\}=6$. Given the same $R$-rep setting, consider a 655 selfish node that has deviated with degree 1 from the correct 656 specification of the protocol. Assuming the current reputa- 657 tion of the node be 6 , then its new reputation after the audit 658 will be: $\rho=\max \{6-2 \cdot 1 \cdot(10-6), 0\}=0$. Note that the set 659 up of the R-rep parameters can yield different results, with 660 varying effects on the nodes' behaviour. In Section 5.4, we 661 will show how the tuning phase of $R A C O O N++$ can support 662 the automatic configuration of these parameters to induce 663 the desired behaviour.

664

\subsection{Selfishness Generation}

The last step of the design phase is the automatic generation 666 of selfish deviations from both the functional specification of 667 the system and the CEM. This is implemented by the Selfish 668 Deviation Generation (SDG) algorithm given in Algorithm 1. 669 The algorithm takes as input a Protocol Automaton and the 670 Selfishness Model SM. Then, it extends the PA with new ele- 671 ments (states, transitions, roles, etc.) representing deviations. 672 Note that the SDG algorithm can generate the deviation 673 types introduced in Section 4.1.2, namely, timeout, subset, and 674 multicast deviations. For brevity, in the pseudo-code we use 675 the notation get(elementId) to refer to the element of the PA 676 to which the elementId identifier is associated. 677

A deviation point is a transition of the PA in which a 678 deviation can take place. To determine if a transition $t \in T 679$ is a deviation point, the SDG algorithm first checks if the 680 SM contains a selfish deviation $d$ that affects $t$ (line 3 in 681 Algorithm 1). Then, it looks for deviation points in lines 4682 (timeout), 8 (subset), and 11 (multicast).

Timeout Deviations. For each deviation point $t \in T$, the 684 algorithm generates a timeout deviation by calling the pro- 685 cedure InjectTimeoutDev (line 5 in Algorithm 1). This proce- 686 dure creates a new final state $s^{\prime}$ and a new abstract 687 transition connecting the source state of $t$ with $s^{\prime}$. 688

Subset Deviations. For each deviation point $t \in T$ triggered 689 by a communication method, SDG checks if the message con- 690 tent $c$ is a collection of data units (line 8). If so, line 9 calls the 691 procedure InjectSubsetDev, which creates new elements to 692 represent the deviation. In particular, the procedure creates 693 a new content $c^{\prime}$ (line 18) that shares the same data type as $c, 694$ but has a shorter length, calculated using d.degree (line 17). 695

Multicast Deviations. For each deviation point $t \in T$ trig- 696 gered by a communication method, the algorithm checks if 697 the receiver of the message sent during $t$ has a cardinality 698 greater than 1 (line 11). If so, line 12 calls the procedure Inject- 699 MulticastDev to create the role $r^{\prime}$ (line 30) with a smaller car- 700 dinality than the correct one (calculated in line 29). 701

Fig. 6 shows the result of executing the SDG algorithm on 702 the Protocol Automaton of Fig. 3. Consider for example 703 state $s_{2}$. In the correct execution of the PA, the role $R_{1}$ sends 704 a response message $\left(g_{3}\right)$ to $r_{0}$. However, if $R_{1}$ is selfish, it 705 may also timeout the protocol or send a message with a 706 smaller payload $\left(g_{3}^{\prime}\right)$.

\section{RACOON++ TUNING PHASE}

The tuning phase of $\mathrm{RACOON++}$ aims at configuring the 709 accountability and reputation mechanisms according to a 710 


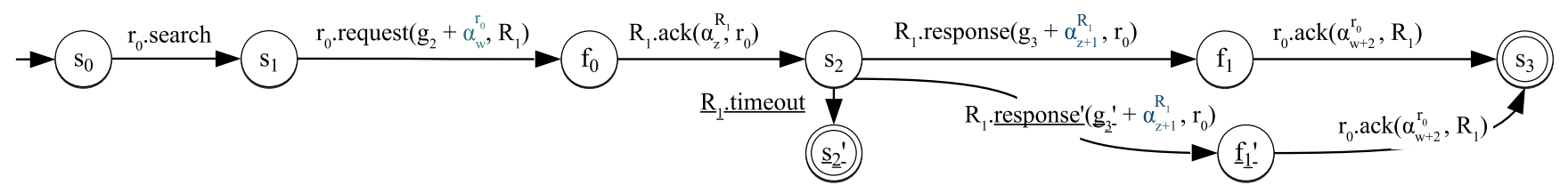

Fig. 6. The protocol automaton of the S-R-R protocol, extended with selfish deviations.

list of design objectives provided by the Designer. Tuning involves an iterative two-step refinement process, which alternates evaluation with the tuning of the configuration parameters. The evaluation involves EGT analysis to study the system dynamics in a given configuration setting. This task is performed by the R-sim simulator integrated into the framework. We have chosen EGT simulations as a modelling tool because in many practical settings the populations of individuals participating in a system evolve towards states of statistical equilibrium. After the evaluation, an exploration algorithm uses the evaluation results to optimise the parameters of the CEM. The tuning process ends after a number of iterations, or when a configuration that satisfies the Designer's objectives is found.

\subsection{Input of the Tuning Phase}

RACOON++ provides a set of selfish-resilience and performance objectives for the cooperative systems designed within its framework. Each objective defines a predicate over a system metric, which can be evaluated by the RACOON++ evaluation tool, i.e., the $R$-sim simulator. The possible predicates are at most and at least. Hereafter, we list some of the application-independent objectives natively supported by $\mathrm{RACOON++}$.

- Cooperation level: the fraction of cooperative nodes in the system;

- Audit precision: the number of correct positive audits divided by the total number of positive audits;

- Audit recall: the number of correct positive audits divided by the number of audits that should have been positive;

- CEM bandwidth overhead: the additional bandwidth consumed by the accountability and reputation mechanisms;

- CEM message overhead: the costs of the accountability and reputation mechanisms in terms of extra messages.

Examples of design objectives are "cooperation level at least 0.8 " and "CEM message overhead at most 0.6 " RACOON ++ allows specifying further objectives on application-specific metrics (e.g., throughput, jitter, anonymity). For each custom objective, the Designer needs to implement the methods to collect and evaluate the related metrics in the evaluation tool.

The second input of the tuning phase is an implementation of the functional specification for the $R$-sim simulator.

\subsection{Evolutionary Game Model}

EGT models how strategic individuals evolve their behaviours by learning and imitating [32]. Similarly to several recent works [27], [28], [29], RACOON++ applies this theoretical framework to model the dynamic behaviour of selfish nodes in a P2P system. The components of an evolutionary game are: (i) a static representation of the system interactions, 762 i.e., the Stage Game; (ii) one or more populations of players; 763 (iii) a function to calculate the utility of a given behaviour; 764 and (iv) the dynamics of the learning and imitation processes. 765 We describe each component separately below.

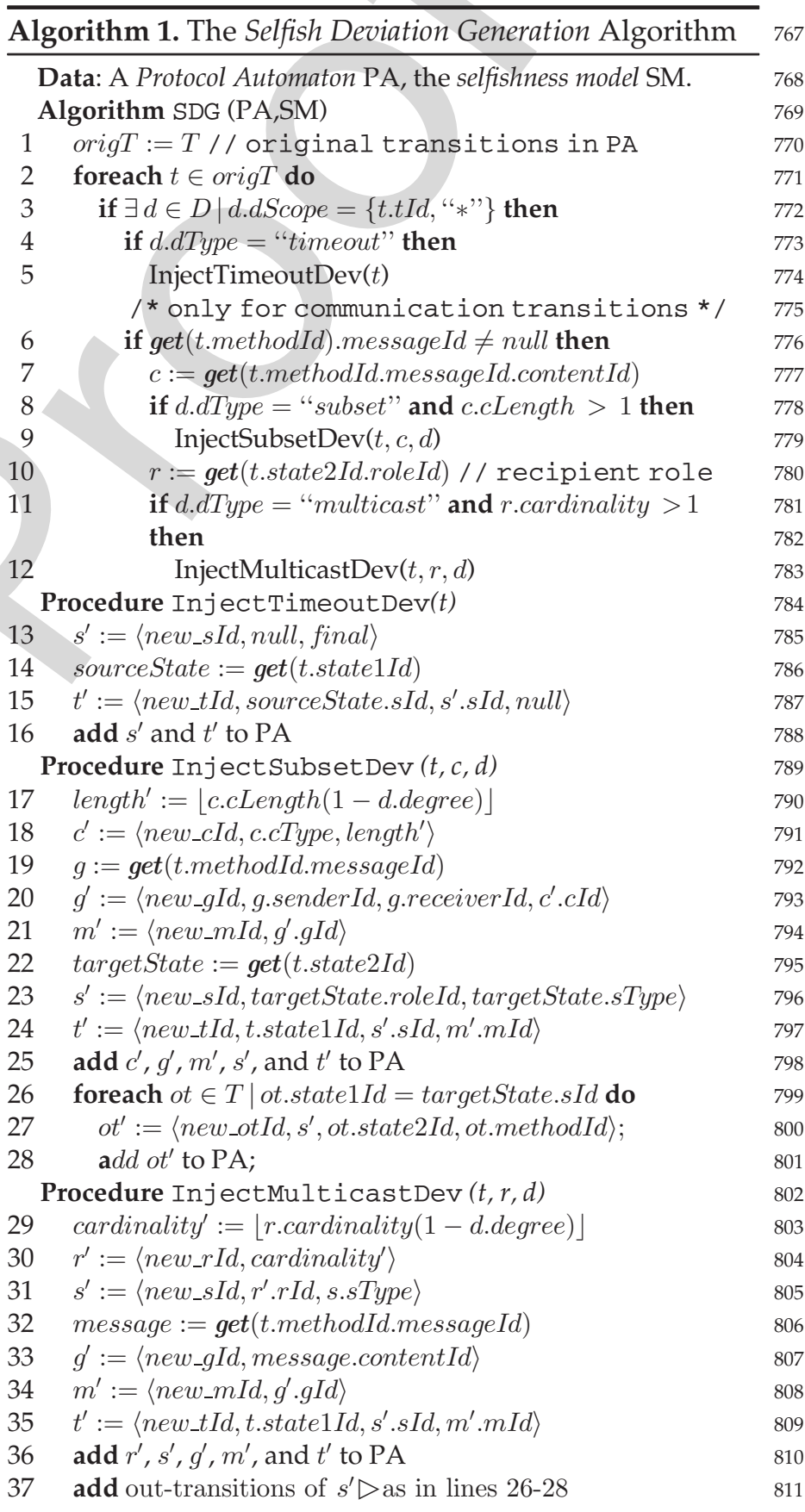

\subsubsection{Stage Game}

Evolutionary games involve the repetition of strategic inter- 813 action between self-interested individuals. We model this 814 


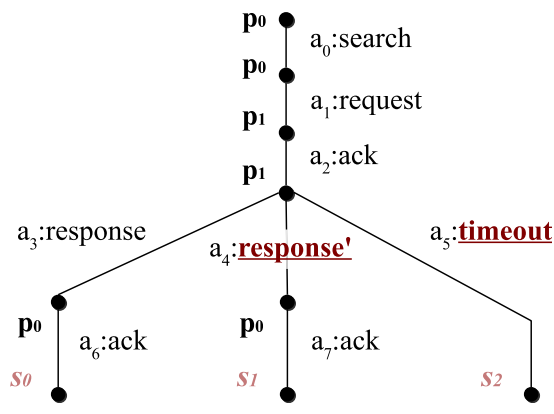

Fig. 7. The SG derived from the S-R-R protocol in Fig. 4. The label besides each decision node indicates the player that takes action at that node. The label on each edge denotes an action along with its corresponding method in the PA. The labels beside each leaf denote the strategy profile of that play.

interaction as a sequential game called the Stage Game, which we represent using the extensive form (or game tree) [31]. Fig. 7 shows the game tree of the stage game derived from the S-R-R protocol illustrated in Fig. 6. $\mathrm{RACOON}++$ provides an automatic tool to create the SG using the information contained in the Extended Specification resulting from the design phase. Specifically, the tool translates the PA included in the Extended Specification into the elements of a stage game as described hereafter.

Players. A player $p$ represents a role of the PA. For example, players $p_{0}$ and $p_{1}$ in Fig. 7 map to roles $r_{0}$ and $R_{1}$ of the S-R-R protocol, respectively. For ease of notation, let $p_{k}$.type refer to the rType of the role mapped by player $p_{k}$.

Nodes. A node of the stage game is derived from a state in the PA, and is labelled with the player who has to take action. A leaf node of the SG corresponds to a final state of the PA, and represents a possible outcome of the stage game. In Fig. 7, each leaf $k$ is labelled with the corresponding outcome $o_{k}$.

Actions. An action is a move of the player in the SG, and is derived from a method in the PA. Note that an edge of the game tree in Fig. 7 corresponds to a transition in the PA. Strategies. A play is a path through the game tree from the root to a leaf. It describes a particular interaction between two (or more) players. The ordered sequence of actions that a player takes in a certain play constitutes her strategy. Consider for instance the left-most play in Fig. 7, which represents the cooperative execution of the S-R-R protocol: Table 1 reports the strategies of players $p_{0}$ and $p_{1}$ to implement it.

\subsubsection{Population of Players}

A population is a group of individuals with common economic and behavioural characteristics. Because of the symmetric nature of cooperative systems, in RACOON++ we consider a single population of nodes, who can play the strategies in the strategy space defined by the stage game. In conformity with the previous works [27] and [28], we

\section{TABLE 1}

The Strategies of the Strategy Profile $s_{0}$, Implementing the Cooperative Execution of the Stage Game in Fig. 7

\begin{tabular}{lc}
\hline Player & Strategy \\
\hline$p_{0}$ & $\left\{a_{0}:\right.$ search, $a_{1}:$ request $, a_{6}:$ ack $\}$ \\
$p_{1}$ & $\left\{a_{2}:\right.$ ack, $a_{3}:$ response $\}$ \\
\hline
\end{tabular}

TABLE 2

The Strategies Implemented in the SG of Fig. 7 When Players $p_{0}$ and $p_{1}$ are from Sub-Populations $\omega_{1}$ and $\omega_{3}$

\begin{tabular}{lcc}
\hline Player & Strategy profile & Strategy \\
\hline$p_{0}$ & $s_{1}$ & $\left\{a_{0}:\right.$ search $, a_{1}:$ request $, a_{6}:$ ack $\}$ \\
$p_{1}$ & $s_{2}$ & $\left\{a_{2}:\right.$ ack, $\left.a_{5}: \underline{\text { timeout }}\right\}$ \\
\hline
\end{tabular}

divide the strategy space into non-overlapping subsets, 850 each representing a distinct combination of behaviours for 851 the nodes (i.e., cooperative, selfishness of a certain type). 852 We call these subsets strategy profiles $s \in \mathcal{S}$. RACOON++ cre- 853 ates a strategy profile $s_{k}$ for each play $k$ of the SG, such that 854 $s_{k}$ includes the strategies carried out by all players partici- 855 pating in that play. Thus, for example, and with reference to 856 Fig. 7, the strategy profile $s_{0}$ represents the behaviour of 857 cooperative nodes and includes the strategies presented in 858 Table 1.

We partition the overall population into sub-populations, 860 so as to establish a one-to-one mapping with the strategy 861 profiles. A sub-population $\omega_{k}$ represents the group of nodes 862 that adopt the behaviour defined by $s_{k}$. In accordance with 863 the EGT model, a member of $\omega_{i}$ participates in the system 864 by repeatedly playing what specified by her strategy profile, 865 regardless of the outcome of the play. However, a member 866 of $\omega_{i}$ can join another sub-population $\omega_{j}$ if she expects to 867 increase his utility by playing $s_{j}$. Thus, the size of a sub- 868 population reflects the success of the associated strategy 869 profile. As the system evolves, the distribution of members 870 across the sub-populations can vary. We call this informa- 871 tion the population state of the system.

\subsubsection{Utility Function}

The utility function of a player assigns a value (i.e., the util- 874 ity) to each outcome of a game. An outcome of the SG 875 depends on the sub-populations of the interacting players, 876 whose strategies determine the particular play that leads to 877 $o$. For example, consider the stage game in Fig. 7, and let 878 players $p_{0}$ and $p_{1}$ be played by members of sub-population 879 $\omega_{1}$ and $\omega_{3}$, respectively. Table 2 lists the planned sequence 880 of actions of the two players. The interaction starts with 881 player $p_{0}$ executing the search transition and then sending a 882 request message to the other player. Player $p_{1}$ will first 883 acknowledge the reception of the message, and then she 884 will terminate the protocol. The interaction described above 885 corresponds to the play $\left\{a_{0}:\right.$ search, $a_{1}$ :request, $a_{2}$ :ack, $a_{5}$ :time- 886 out $\}$ in Fig. 7, which leads to the outcome induced by the 887 strategy profile $s_{2}$. The outcomes of a stage game describe 888 the interaction between every possible combination of play- 889 ers from different sub-populations.

In $R A C O O N++$, the utility received from playing a stage 891 game has two terms: the protocol payoff, and the incentives 892 introduced by the CEM. The protocol payoff $\gamma_{j}$ evaluates 893 the costs and benefits of a player when the outcome of SG is 894 $o_{j}$. To calculate this value, $R A C O O N++$ evaluates the valua- 895 tion elements defined in the selfishness model by the 896 Designer (see Section 4.1.2). Let us illustrate the procedure 897 to evaluate the protocol payoff $\gamma_{0}$ in the stage game of 898 Fig. 7, in the case of interaction between members of the 899 cooperative sub-population $\omega_{0}$. Consider the following 900 valuations associated to role $r_{0}$ and, thus, to player $p_{0}: 901$ 
$\left\langle v_{0}\right.$, search, $\left.r_{0}, 10,1\right\rangle$ and $\left\langle v_{1}, g_{3}, r_{0}, 3,1\right\rangle$, which refer to the $a_{0}$ : search and $a_{3}$ :response edges in Fig. 7, respectively. Let the content $c \in C$ carried by message $g_{3}$ be a list of 10 data units. Then, the protocol payoff of player $p_{0}$ is

$$
\begin{aligned}
\gamma_{0}\left(p_{0}\right) & =v\left(v_{0}\right)+v\left(v_{1}\right) \\
& =9+2 \cdot r_{0} . \text { cardinality } \cdot \text { c.cLength }=29 .
\end{aligned}
$$

The protocol payoff is the expected utility that would be received if no incentives for cooperation were attached to the system. However, the CEM used in RACOON++ establishes that the ability of a player to obtain a requested service is proportional to her reputation value (see Section 4.2). Thus, the utility $u_{j} \in \mathbb{R}$ obtained by a player $p_{i}$ depends on whether she plays as a service requester in the stage game. Formally

$$
u_{j}\left(p_{i}\right)=\left\{\begin{array}{ll}
\gamma_{j}\left(p_{i}\right) \cdot \varrho\left(p_{i}\right) & \text { if } p_{i} . \text { type }=\text { "requester" } \\
\gamma_{j}\left(p_{i}\right) & \text { otherwise }
\end{array},\right.
$$

where the function $\varrho: \mathcal{P} \rightarrow[0,1]$ determines the probability that player $p_{i} \in \mathcal{P}$ will receive the protocol payoff, calculated as the reputation of $p_{i}$ divided by the upper bound $\rho_{\max }$ of reputation values. Following on the previous example, let the reputation mechanism allow values between 0 and 10 , and let the requester player $p_{0}$ have reputation 6 . Then, her utility can be calculated as

$$
u_{0}\left(p_{0}\right)=\gamma_{0}\left(p_{0}\right) \cdot \rho\left(p_{0}\right)=29 \cdot 0.6 \simeq 17.4
$$

\subsubsection{Evolutionary Dynamics}

A common assumption in classical game theory is that players have the information and skills to assess and choose the best strategy to play in the current system's state [31]. However, as other works have highlighted [27], [28], [29], this assumption places a heavy burden on nodes' computational and communication capabilities, which is infeasible in most real-world cooperative systems. On the contrary, EGT assumes that players are neither perfectly rational nor fully informed about all the possible strategies, but tend to implement the most remunerative strategy through learning and imitation [32].

In $\mathrm{RACOON++}$, each node monitors the utility it has obtained for playing the strategy profile of its subpopulation. If the utility decreases for more than a given number of consecutive observations, or if a specified time has elapsed, then the node will look for a fitter subpopulation to join. The accountability audits of $R$-acc provide the means to learn what are the fittest sub-populations in the system. More precisely, we assume that a witness can infer the sub-population and the utility of a node by auditing its logs, as the recorded actions can be traced back to a particular strategy profile (the space of strategy profiles, as well as the costs and benefits of each action, are common knowledge to all nodes, because we assume a single population). After an audit, the witness compares its own utility against that of the monitored node. If the witness has a lower utility, it will join the sub-population of the monitored node with a given probability [29]. This probability determines the evolution rate: the smaller its value, the slower the fittest sub-population in the system increases.

\subsection{Game-Based Evaluation}

The game-based evaluation step evaluates a configuration 960 setting for the CEM. To this end, RACOON++ first creates 961 an evolutionary game model of the system and then it simu- 962 lates the game dynamics using the RACOON++ evaluation 963 tool $R$-sim. The simulation results indicate whether the eval- 964 uated CEM configuration has satisfied the list of design 965 objectives set by the Designer or not.

The RACOON++ simulation framework, $R$-sim, uses the 967 evolutionary game model of the cooperative system to sim- 968 ulate the system dynamics in the candidate configuration 969 setting. The networking environment of $R$-sim consists of 970 independent nodes that are able to send a message to any 971 other node, provided that the address of the target node is 972 known. Communication is assumed to be subject to arbi- 973 trary message loss, controlled by a probability parameter. 974 Nodes can leave and join the network at any time. The sim- 975 ulation engine of $R$-sim supports a cycle-based model, in 976 which time is structured into rounds. At each round, each 977 node plays a certain strategy of the SG, according to the evo- 978 lutionary dynamics described in Section 5.2. During the 979 simulation $R$-sim collects statistics about such dynamics, to 980 evaluate the design objectives.

In contrast with $R A C O O N$, which includes a custom-built 982 simulator for cooperative systems [19], RACOON++ relies 983 on the state-of-the-art PeerSim simulator [10], thereby 984 improving the usability, accuracy and performance of the 985 framework. We have chosen PeerSim among other simula- 986 tion tools (see [39] for a comprehensive review) because: (1) 987 it meets the requirements of scalability and dynamicity 988 imposed by the evolutionary model; (2) it supports integra- 989 tion with RACOON++ thanks to its modular architecture; (3) 990 it is an active project, with a good developer community and 991 support. R-sim exploits the modular architecture of PeerSim 992 extending it with new components to develop, simulate and 993 evaluate the cooperative system resulting from the design 994 phase of the RACOON++ framework. Also, R-sim includes a 995 reference implementation of the accountability and reputa- 996 tion systems used by $R A C O O N++$, along with an intuitive 997 API to simulate their calls. The Designer can use these facili- 998 ties to implement the functional specification of his system 999 for PeerSim. To the best of our knowledge, $R$-sim is the only 1000 available software tool for the dynamic simulation of selfish 1001 and strategic behaviours in distributed systems.

Other important $R$-sim parameters are listed below:

- Network: the network size; the message loss rate.

- Evolutionary game model: the initial population state 1005 (e.g., equal-sized sub-populations, majority of coop- 1006 erative nodes); the probability to join a fitter sub- 1007 population.

- Monitoring: the duration of a simulation; the fre- 1009 quency and the types of statistics to collect (e.g., 1010 nodes' payoffs, amount of messages exchanged, 1011 audit results).

\subsection{Design Space Exploration}

The output of the RACOON++ framework is the design and 1014 configuration of a cooperative system that achieves the 1015 design objectives set by the Designer. Thus far, we have 1016 described how RACOON++ fosters cooperation using 1017 
accountability and reputation mechanisms (Section 4.2), and how it evaluates the system performance using EGT and simulation (Section 5.3). The last step of the framework relies on the evaluation results to tune the configuration parameters of the CEM, aiming to achieve the desired design objectives. A configuration candidate is an assignment of the $R$-acc and $R$-rep parameters, i.e., the size of the witness set, the audit period, the audit probability, the degree of punishment, and the degree of reward.

The exploration is an iterative process, which generates new candidates based on the evaluation of the previous ones until a configuration is found that satisfies all the Designer's objectives. If no feasible solution is found after a pre-defined number of iterations (e.g., because the objectives were contradictory or too demanding), the framework stops the search, asking the Designer to improve the design manually or to relax the design objectives.

RACOON++ explores the configuration space using a greedy constraint-satisfaction algorithm, which is guided by a set of observations derived from an empirical analysis of the CEM parameters and their impact on the design objectives natively supported by $\mathrm{RACOON++.}{ }^{3}$ For instance, we observed that the higher the number of witnesses, the higher the CEM bandwidth overhead, because each witness increases the amount of log transmissions and checking. As another example, we observed that the shorter the audit period, the higher the cooperation level, because selfish nodes are detected earlier and punished more often. The exploration algorithm relies on these observations to generate the next configuration candidate. For instance, if the evaluation of a given configuration results in a bandwidth overhead larger than what required by a design objective, the exploration algorithm will not generate configuration candidates with a greater number of witnesses. If no guidelines are available for updating a particular configuration, the exploration algorithm will create a random configuration candidate. In order to avoid the re-exploration of the regions of the configuration space, the algorithm records the previously generated candidates.

\section{USING THE RACOON++ FRAMEWORK}

$\mathrm{RACOON++}$ is provided as a Java program, which is released under a free software licence and is publicly available [33]. In the previous sections, we described the main steps and building blocks of the framework. Now we turn our attention to how RACOON++ is used by the Designer.

The first step for the Designer is to decide what parts of the system should be included in the RACOON++ functional specification (i.e., the Protocol Automata). The selected parts should fulfil two criteria. On the one hand, these parts should represent system functionalities that are sensitive to selfish behaviours-specifically, to the deviation types described in Section 4.1.2. On the other hand, the selected parts should involve actions that can be observed by other nodes (e.g., a message exchange), to allow accountability audits [12], [13].

3. The analysis involved the systematic evaluation of 250 configuration candidates in three cooperative systems (i.e., the ones considered for evaluating our work) for a total of 750 experiments.
Then, the Designer inputs the functional specification, 1073 along with the selfishness model to study (Section 4.1.2) and 1074 the design objectives to achieve (Section 5.1), to the frame- 1075 work. In RACOON++, these specifications are encoded in 1076 an XML-based format, and are provided as a single XML 1077 document. ${ }^{4}$

To evaluate a configuration setting for the CEM, 1079 $R A C O O N++$ simulates the system behaviour using the inte- 1080 grated simulation framework R-sim, based on the PeerSim 1081 simulator. To this end, the Designer has to produce a Java 1082 implementation of the cooperative system, notably of its 1083 functional specification. $R$-sim facilitates this task by provid- 1084 ing a set of ready-to-use components and an intuitive 1085 API for interfacing a standard PeerSim protocol with the 1086 RACOON++ models and functionalities. In particular, 1087 the framework includes an implementation of the CEM, the 1088 algorithms to simulate the behaviour of selfish nodes, and 1089 monitors to assess application-independent system perfor- 1090 mance (e.g., audit precision and recall, bandwidth over- 1091 head). These software facilities reduce the number of 1092 functionalities to code, allowing the Designer to focus only 1093 on implementing the application specific parts of her sys- 1094 tem, such as the code to implement the correct execution of 1095 the protocol and the selfish deviations from it.

1096

Once all the inputs have been defined, the Designer can 1097 run the RACOON++ framework and wait for the result of 1098 its design and tuning phases (Fig. 1).

\section{Evaluation}

In this section, we demonstrate the benefits of using 1101 $R A C O O N++$ to design selfishness-resilient cooperative sys- 1102 tems. First, we introduce the three use cases considered in 1103 the evaluation, namely, a live-streaming protocol, a load 1104 balancing protocol, and an anonymous communication sys- 1105 tem. Second, we assess the effort required by a Designer to 1106 specify and implement the use cases. Third, we evaluate the 1107 capability of RACOON++ to auto-configure the CEM, by 1108 measuring the time needed to find a satisfactory configura- 1109 tion in 90 different scenarios. Then, we evaluate the effec- 1110 tiveness of the RACOON++ cooperation enforcement 1111 mechanisms in withstanding the impact of selfish nodes on 1112 a set of performance objectives. Finally, we compare the per- 1113 formance of the CEM's accountability mechanism with Full- 1114 Review, showing that $R$-acc achieves better results while 1115 imposing less overhead.

The implementation of the use cases, as well as the con- 1117 figuration files related to the experiments reported in this 1118 section, can be downloaded from the project website [33].

\subsection{Use Cases}

We consider the following use cases.

Live Streaming. A P2P live streaming system consists of a 1122 source node that disseminates video chunks to a set of 1123 nodes over a network. Periodically, each node sends the 1124 chunks it has received to a set of randomly chosen partners 1125 and asks them for the chunks they are missing. Each chunk 1126 is associated with a playback deadline, which, if missed, 1127 would render a chunk unusable and the corresponding 1128

4. The XML Schema for this document can be found in [33] 


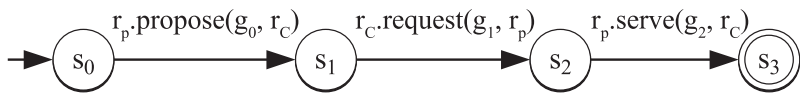

Fig. 8. The PA of the live streaming protocol [14].

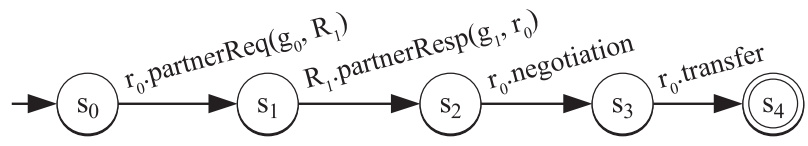

Fig. 9. The PA of the load balancing protocol [10].

portion of the video unplayable. For the chunk exchange, we use the gossip-based live streaming protocol studied by Guerraoui et al. [14]. Load Balancing. The heterogeneity of nodes and the high dynamics of P2P systems can lead to a load imbalance. ${ }^{5}$ We assume a P2P system in which nodes are allowed to transfer all or a portion of their load among themselves. The goal of a load balancing protocol is to regulate these transfers in a way that evenly distributes the load among nodes, to optimise the use of node capabilities. The load balancing protocol considered as a use case is the one proposed by Jelasity et al. [10].

Anonymous Communication. This system is based on a simplified version of the Onion Routing protocol for communication channel anonymity [15]. In Onion Routing, when a source node wants to send a message to a destination node, the source node builds a circuit of voluntary relay nodes. Circuits are updated periodically, and relays can participate in multiple circuits at the same time. To protect a message, the source encrypts it with the public key of the destination. Furthermore, to protect the communication channel, the source uses the public key of each relay node in the circuit to encrypt the address of the next relay node. The resulting message is called an onion. A relay uses its private key to decrypt one layer of the onion and contributes some of its bandwidth to forward the resulting message to the next relay until the message eventually reaches its destination.

\subsection{Design and Development Effort}

To show the benefits of using RACOON++ in terms of design and development effort, we present the operations that allow the Designer to specify, develop, and test the use cases.

To begin, the Designer specifies the communication protocols (i.e., Protocol Automata) to be included in the functional specification of the system. Figs. 8, 9, and 10 illustrate the Protocol Automata defined for our use cases. The live streaming protocol (see Fig. 8) involves two roles and three protocol steps: the provider $r_{p}$ proposes the set of chunks it has received to a set of consumers $r_{C}$, which in turn request the chunks they need. The protocol ends when $r_{p}$ sends the requested chunks to $r_{C}$. In the load balancing protocol (see Fig. 9) each node starts with a certain amount of load. Periodically, each node $r_{0}$ is allowed to transfer all or a portion of its load to one of its neighbours $R_{1}$, after a negotiation step. The negotiation is based on locally available information, obtained from past interactions or sample observations [10]. Lastly, in the anonymous communication protocol, every time a relay $r_{r}$ receives an onion message from its predecessors $\left(r_{P}\right)$ in the

5. The load can be measured in terms of different metrics, such as the number of queries received per time unit.

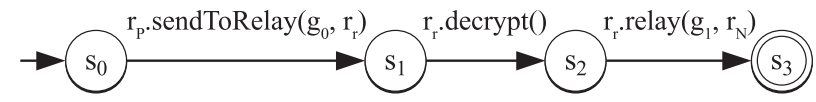

Fig. 10. The PA of the anonymous communication protocol.

TABLE 3

Lines of Code Needed for the Use Cases

\begin{tabular}{lcccc}
\hline & Specification & \multicolumn{3}{c}{ R-sim Program $^{\mathrm{a}}$} \\
\cline { 2 - 5 } & & Std & RS & TOT \\
\hline Live Streaming protocol & 51 & 384 & 28 & 444 \\
Load Balancing protocol & 48 & 232 & 28 & 290 \\
Anonymous Comm. protocol & 48 & 212 & 23 & 289 \\
\hline
\end{tabular}

Std $=$ standard operation, $R S=\mathrm{R}$-sim functionalities, $T O T=S t d+R S$.

circuit, $r_{r}$ decrypts the external layer of the onion, and for- 1175 wards the resulting onion to the next hops $r_{N}$ in the circuit. If 1176 $r_{r}$ is the final destination of the onion, then the protocol will 1177 end after the decrypt transition (state $s_{2}$ of Fig. 10).

Once the Designer has provided the functional specifica- 1179 tion of the system, she defines the selfishness model. For 1180 example, consider the anonymous communication protocol. 1181 A selfish relay $r_{r}$ that wants to save bandwidth may strate- 1182 gically avoid to forward onions that are not intended for 1183 itself. Concretely, $r_{r}$ could avoid to relay any onion to its 1184 successors (timeout deviation) or relay onions only to a sub- 1185 set of them (multicast deviation). As another example, con- 1186 sider a selfish provider $r_{p}$ that wants to participate in the 1187 live streaming protocol but limits its bandwidth consump- 1188 tion. A possible strategy for $r_{p}$ is to propose fewer chunks 1189 than it has available (subset deviation), or send proposals to 1190 only a subset of its neighbours (muticast deviation), in such 1191 a way as to reduce the number of chunks that could be 1192 requested.

Finally, the Designer provides $R A C O O N++$ with a list of 1194 design objectives that the system must satisfy. Recall from 1195 Section 5.3 that an objective can be application-independent 1196 or application-specific. Examples of application-specific 1197 objectives related to our use cases are (i) a load distribution 1198 with a Coefficient of Variation (CoV) close to zero, (ii) a low 1199 fraction of onions that do not reach their final destination, or 1200 (iii) a low fraction of video chunks that are not played in time. 1201

The Designer provides the RACOON++ specification 1202 inputs as an XML document. The "Specification" column of 1203 Table 3 illustrates the conciseness of the XML representation 1204 of the inputs, showing that the full specification of a use 1205 case does not require many Lines of Code (LoC).

The $R A C O O N++$ framework requires the Designer to 1207 implement the functional specification of the system in the 1208 $R$-sim simulator. The "R-sim Program" columns of Table 31209 shows the LoC of the use cases' implementations, distin- 1210 guishing the LoC needed to implement the standard opera- 1211 tion ("Std" column) from those introduced to invoke the 1212 $R$-sim functionalities ("RS" column). The results show that 1213 the software facilities provided by $R$-sim allow adapting a 1214 system implementation to be used in RACOON++ without 1215 significant coding effort. More precisely, the RS LoC are in 1216 the range 6.3-9.6 percent of the total implementation code, 1217 which appears reasonable as it corresponds to only 28 addi- 1218 tional LoC, at most. 


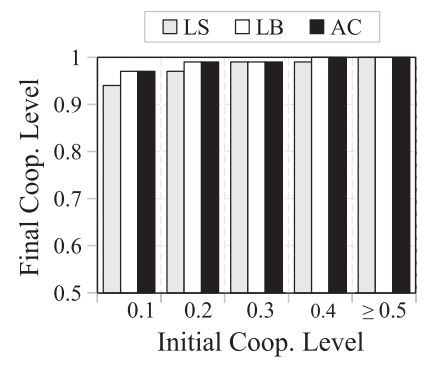

(a) Cooperation Level

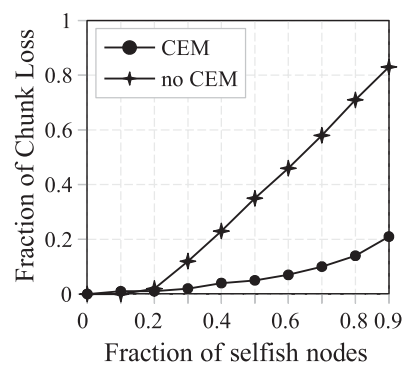

(b) Chunk Loss in LS

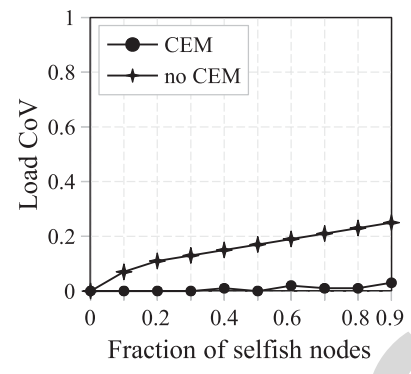

(c) Load $\mathrm{CoV}$ in $\mathrm{LB}$

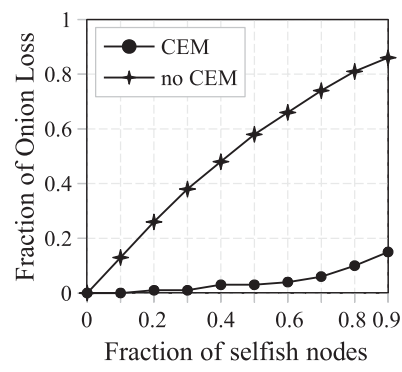

(d) Onion Loss in AC

Fig. 11. Cooperation levels (a) and application-specific performance of the Live Streaming (LS) (b), Load Balancing (LB) (c), and Anonymous Communication (AC) (c) use cases, when varying the initial fraction of selfish nodes.

\subsection{Meeting Design Objectives Using RACOON++}

To evaluate the capability of $R A C O O N++$ to find a satisfactory configuration for its cooperation enforcement mechanisms, we performed the following experiment. First, we defined 30 different scenarios for each use case, for a total of 90 scenarios, where a scenario is a unique combination of design objectives, system parameters (e.g., number of nodes, message loss rate), application-specific parameters (e.g., playback deadline, length of a circuit of relays, initial distribution of loads), and fraction of selfish nodes in the system. ${ }^{6}$ Second, we used $R A C O O N++$ to find a satisfactory configuration for each scenario, while measuring the number of tested configurations and the duration of the process. In the experiment, $\mathrm{RACOON++}$ tests an average of 7 configurations before finding a satisfactory one (median 4, range 1-56). The process takes less than $18 \mathrm{~min}$ on average to complete (median $6 \mathrm{~min}$, range $10 \mathrm{~s}-208 \mathrm{~min}){ }^{7}$ which we consider reasonable, as $R A C O O N++$ runs offline at design time.

Overall, the tuning process failed to meet all the design objectives in only three scenarios out of 90, which we consider as an acceptable result. The failures were due to too hard constraints on the efficiency and effectiveness of the CEM, which were expressed as cost overhead and custom performance objectives (such as low video chunk loss rate), respectively. In these cases, $R A C O O N++$ returns to the Designer the tested configuration that has obtained the best performance in terms of the design objectives. If not satisfied with this outcome, the Designer can either relax the performance requirements or optimise some applicationspecific operation or parameter.

\subsection{RACOON++ Effectiveness}

In this section, we show that the cooperative systems designed using $R A C O O N++$ can effectively achieve cooperation as well as application-specific objectives in the presence of an increasing proportion of selfish nodes. To this end, we evaluated three scenarios per use case, which were randomly selected from the scenarios generated for the previous experiment.

In the first experiment, we assess the effectiveness of the $\mathrm{CEM}$ in fostering cooperation in the tested systems. The experiment consists of a set of simulations, which monitor the dynamics of 2,000 nodes for 3,000 simulation cycles. We

6. For reasons of space, the full setting for this and the following experiments is not reported here, but have been made available on the project website [33].

7. Measures made on a $2.8 \mathrm{GHz}$ machine with $8 \mathrm{~GB}$ of RAM. initialize each simulation with an increasing proportion of 1262 cooperative nodes (from 0.1 to 1 ), and we measure the coop- 1263 eration level achieved at the end of the simulation. For each 1264 use case, we calculated the median result from the three sce- 1265 narios. Results in Fig. 11a show that the CEM succeeds in 1266 making the nodes behave cooperatively in all use cases. 1267 Even the worst result (in the live streaming use case) shows 1268 a dramatic increase of the cooperation level, from 0.1 to 0.94. 1269

We now focus on the correlation between cooperation 1270 level and application-specific performance. Figs. 11b, 11c, 1271 and $11 \mathrm{~d}$ present the median results of our evaluation for the 1272 three use cases.

The figures display a curve showing the impact of selfish 1274 nodes when no cooperation enforcement mechanism is 1275 adopted (curve no CEM), and another curve for the results 1276 obtained when using RACOON++ (curve CEM). For exam- 1277 ple, Fig. 11d shows that without any mechanism to prevent 1278 selfishness the fraction of onions that do not reach destina- 1279 tion in the anonymous communication use case increases 1280 linearly with the number of selfish nodes in the system and 1281 reaches very high values (e.g., 40 percent of selfish nodes 1282 leads to a loss of almost half of the transmitted onions, 1283 thereby making the system ineffective in practice). Similar 1284 conclusions hold for the number of chunks in the live 1285 streaming use case Fig. 11b. The initial cooperation level 1286 also has an impact on the performance of the load balancing 1287 protocol, which we measured in terms of CoV of the load 1288 distribution (the lower the $\mathrm{CoV}$, the better the performance). 1289 As we can observe in Fig. 11b, when no mechanism to foster 1290 cooperation is in place the CoV increases with the number 1291 of nodes that refuse to participate in the balancing protocol. 1292 In contrast, the results achieved by the systems designed 1293 using RACOON++ show that the CEM can withstand the 1294 impact of large populations of selfish nodes.

\subsection{RACOON++ versus FullReview}

In this section, we present the benefits of using the 1297 RACOON++ CEM instead of the original FullReview proto- 1298 cols [13]. The main differences between these mechanisms, 1299 already discussed in Section 4.2, are (i) the approach to pun- 1300 ishing selfish and suspect nodes, which is more tolerant in 1301 the CEM, (ii) the possibility in R-acc to control the probabil- 1302 ity of auditing other nodes, (iii) the dissemination of proofs 1303 of misbehaviour in the system, which in RACOON++ is 1304 realized by R-rep. To compare the performance of the 1305 RACOON++ CEM and of FullReview in our use cases, we 1306 initialized the tested systems with a scenario randomly cho- 1307 sen from the set created for the previous experiment. Then, 1308 


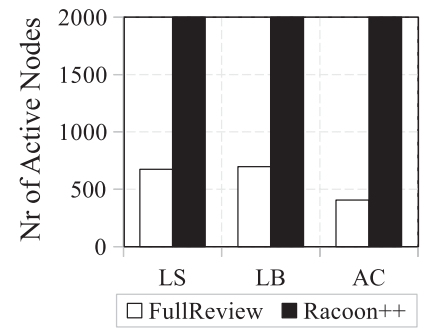

(a) Active Nodes

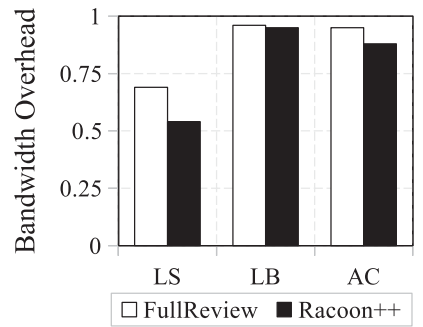

(b) Bandwidth Overhead

Fig. 12. Performance comparisons between FullReview and RACOON+ + CEM in the Live Streaming (LS), Load Balancing (LB), and Anonymous Communication (AC) use cases.

we performed two sets of simulations for each system. In one set we used the RACOON++ CEM to foster cooperation, and in the other set we used FullReview. Both the CEM and FullReview were optimised for the scenario. In particular, the CEM was automatically configured by the RACOON++ tuning phase, whereas FullReview was tuned manually.

The first important benefit of using CEM is shown in Fig. 12a, which represents the fraction of nodes that are participating in the cooperative system at the end of the simulation. This figure readily illustrates the opposite approaches adopted by RACOON++ and FullReview to deal with selfishness: RACOON++ aims to motivate selfish nodes to change their strategy and behave cooperatively, while FullReview operates by isolating non-cooperative nodes. We advocate our approach as the most appropriate for cooperative systems, for two reasons. First, it takes into account the high heterogeneity of nodes and allows low-resource nodes to occasionally behave selfishly because of resource shortages (e.g., low battery in mobile devices). Second, it fits better with the cooperative design principles, which are based on participation and inclusion rather than on punitive restrictions.

On the performance side, Fig. 12b shows that the CEM of $\mathrm{RACOON++}$ can decrease the bandwidth overhead in the tested system, notably by 22 percent in the live streaming use case. This is mainly due to the replacement of the evidence transfer protocol of FullReview with a lightweight reputation system, in which reputation values are exchanged by piggybacking on the accountability protocols messages. Also, R-acc allows probabilistic audits, which further reduces the traffic and computation overhead associated with the audit activities.

As shown in earlier work [19], FullReview is very sensitive to message loss, which can significantly increase the number of suspect nodes, and might even lead to the

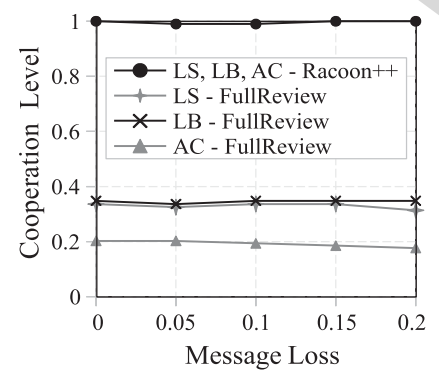

(a) Cooperation Level

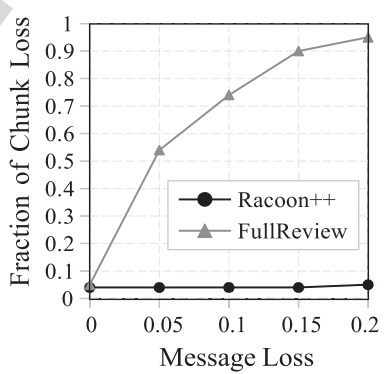

(b) Chunk Loss in LS wrongful eviction of a correct node. We evaluated the 1344 robustness of the RACOON++ CEM against message loss by 1345 assessing the performance of the tested systems when run- 1346 ning over an unreliable network with up to 20 percent mes- 1347 sage loss. Fig. 13a illustrates the cooperation levels achieved 1348 by the tested systems at the end of the simulations when 1349 using the RACOON++ CEM and FullReview. The curves 1350 show that message loss has a small impact on the coopera- 1351 tion, due to the mitigating effect of the challenge/response 1352 protocol used by both mechanisms (see Section 4.2). Notice 1353 that the FullReview curves in Fig. 13a confirm what already 1354 discussed for Fig. 12a, that is the dramatic decrease of active 1355 nodes because of the extreme punishment enforced by the 1356 accountability mechanism. Such performance degradation 1357 is much more severe for application-specific objectives, as 1358 can be observed in Figs. 13b, 13c, and 13d. The main reason 1359 is the FullReview suspicion mechanism, which prevents a 1360 suspect node from interacting with others. Because tempo- 1361 rary message loss can trigger node suspicion, the larger the 1362 message loss rate, the longer a node could be stuck in a sus- 1363 pect state. Conversely, in the RACOON++ CEM, a suspect 1364 node can continue to interact with other nodes, though with 1365 a lower probability. This gives the suspect node more 1366 opportunities to get out of the suspect state by behaving 1367 cooperatively, which is also beneficial for the system. The 1368 Racoon++ curves in Figs. 13b, 13c, and 13d demonstrate that 1369 this simple strategy is enough to guarantee resilience from 1370 selfish nodes while being tolerant to message loss.

\section{Conclusions}

In this paper we presented $R A C O O N++$, a model-based 1373 framework for designing, configuring, and testing coopera- 1374 tive systems that are resilient to selfish nodes. RACOON++ 1375 relies on accountability and reputation mechanisms to 1376 enforce cooperation among selfish nodes. Using a combina- 1377 tion of simulation and Evolutionary Game Theory, 1378 RACOON++ automatically configures these mechanisms in 1379 a way that meets a set of design objectives specified by the 1380 system designer. We illustrated the benefits of using 1381 RACOON++ by designing a P2P live streaming system, a 1382 load balancing protocol, and an anonymous communication 1383 system. The evaluation of the use cases, performed using 1384 the state-of-the-art simulator PeerSim, shows that the 1385 cooperative systems designed using RACOON++ achieve 1386 selfishness-resilience and high performance. The RACOON 1387 ++ framework is provided as a Java program, and is freely 1388 available for download [33].

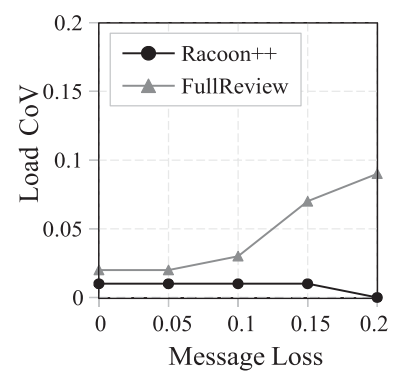

(c) $\mathrm{CoV}$ of load in $\mathrm{LB}$

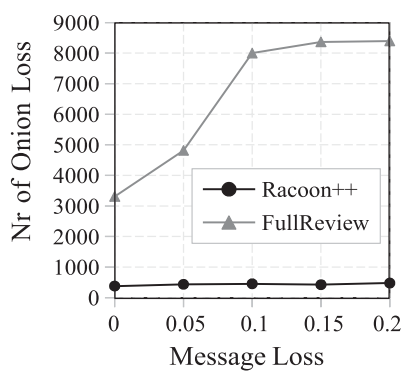

(d) Onion Loss in AC

Fig. 13. Experiment results with different proportions of message loss. 
Our future work includes the integration of a domainspecific language into $R A C O O N++$ to specify more complex selfish behaviours, such as the one we proposed in [42], and the investigation of other mechanisms to foster cooperation (e.g., decentralised credit-based systems).

\section{REFERENCES}

[1] Cisco Systems, "Cisco Visual Networking Index: Forecast and Methodology, 2015-2020," A Cisco White Paper, 2016.

[2] X. Liu, et al., "A case for a coordinated internet video control plane," in Proc. ACM SIGCOMM Conf. Appl. Technol. Archit. Protocols Comput. Commun., 2012, pp. 359-370.

[3] N. Anjum, D. Karamshuk, M. Shikh-Bahaei, and N. Sastry, "Survey on peer-assisted content delivery networks," Comput. Netw., vol. 116, pp. 79-95, 2017.

[4] M. Swan, Blockchain: Blueprint for a New Economy. Sebastopol, CA, USA: O'Reilly Media, Inc., 2015.

[5] R. Want, B. N. Schilit, and S. Jenson, "Enabling the internet of things," IEEE Comput., vol. 48, no. 1, pp. 28-35, Jan. 2015.

[6] G. Ziccardi, Resistance, Liberation Technology and Human Rights in the Digital Age. Berlin, Germany: Springer, 2012.

[7] I. Cunha, E. C. Miguel, M. V. Rocha, J. Oliveira, A. Vieira, and S. Campos "Can peer-to-peer live streaming systems coexist with free riders?" in Proc. IEEE 30th Int. Conf. Peer-to-Peer Comput., 2013, pp. 1-5

[8] M. Zghaibeh and H. C. Fotios, "Revisiting free riding and the Titfor-Tat in BitTorrent: A measurement study," Peer-to-Peer Netw. Appl., vol. 1, no. 2, pp. 162-173, 2008.

[9] D. Hughes, G. Coulson, and J. Walkerdine, "Free riding on Gnutella revisited: The bell tolls?" IEEE Distrib. Syst. Online, vol. 6, no. 6, Jun. 2005, Art. no. 1.

[10] M. Jelasity, A. Montresor, and O. Babaoglu, "A modular paradigm for building self-organizing peer-to-peer applications," in Proc. Int. Workshop Eng. Self-Organizing Appl., 2003, pp. 265-282.

[11] B. Cohen, "Incentives build robustness in BitTorrent," in Proc. Int Workshop Econ. Peer-to-Peer Syst., 2003, vol. 6, pp. 68-72.

[12] A. Haeberlen, P. Kouznetsov, and P. Druschel, "PeerReview: Practical accountability for distributed systems," in Proc. 21st ACM SIGOPS Symp. Operating Syst. Principles, 2007, pp. $175-188$.

[13] A. Diarra, S. Ben Mokhtar, P. L. Aublin, and V. Quéma, "FullReview: Practical accountability in presence of selfish nodes," in Proc. IEEE 33rd Int. Symp. Reliable Distrib. Syst., 2014, pp. 271-280.

[14] R. Guerraoui, K. Huguenin, A-M. Kermarrec, M. Monod, and S. Prusty, "LiFTinG: Lightweight freerider-tracking in gossip," in Proc. ACM/IFIP/USENIX Int. Conf. Distrib. Syst. Platforms Open Distrib. Process., 2010, pp. 313-333.

[15] D. Goldschlag, M. Reed, and P. Syverson, "Onion routing," Commun. ACM, vol. 42, no. 2, pp. 39-41, 1999

[16] I. Abraham, D. Dolev, R. Gonen, and J. Halpern, "Distributed computing meets game theory: Robust mechanisms for rational secret sharing and multiparty computation," in Proc. 25th Annu. ACM Symp. Principles Distrib. Comput., 2006, pp. 53-62.

[17] J. Kats, "Bridging game theory and cryptography: Recent results and future directions," in Theory of Cryptography. Berlin, Germany: Springer, 2008

[18] J. Freudiger, M. H. Manshaei, J-P. Hubaux, and D. C. Parkes, "On non-cooperative location privacy: A game-theoretic analysis," in Proc. 16th ACM Conf. Comput. Commun. Secur., 2009, pp. 324-337.

[19] G. Lena Cota, et al., "A framework for the design configuration of accountable selfish-resilient peer-to-peer systems," in Proc. IEEE 34th Int. Symp. Reliable Distrib. Syst., 2015, pp. 276-285.

[20] L. Buttyán, L. Dóra, M. Félegyházi, and I. Vajda, "Barter trade improves message delivery in opportunistic networks," Ad Hoc Netw., vol. 8, no. 1, pp. 1-14, 2010.

[21] R. Trestian, O. Ormond, and G. M. Muntean, "Game theory-based network selection: Solutions and challenges," IEEE Commun. Surveys Tuts., vol. 14, no. 4, pp. 1212-1231, Oct.-Dec. 2012.

[22] A. S. Aiyer, L. Alvisi, A. Clement, M. Dahlin, J. P. Martin, and C. Porth, "BAR fault tolerance for cooperative services," in Proc. 20th ACM SIGOPS Symp. Operating Syst. Principles, 2005, pp. 45-58.
[23] S. Ben Mokhtar, G. Berthou, A. Diarra, V. Quéma, and A. Shoker, 1463 "RAC: A freerider-resilient, scalable, anonymous communication 1464 protocol," in Proc. IEEE 33rd Int. Conf. Distrib. Comput. Syst., 2013, 1465 pp. 520-529.

[24] H. C. Li, et al., "FlightPath: Obedience versus choice in coopera- 1467 tive services," in Proc. 8th USENIX Symp. Operating Syst. Des. 1468 Implementation, 2008, pp. 355-368.

25] H. C. Li, et al "BAR gossip" in Proc 7th USENIX Symp. Operating 1470 Syst. Des. Implementation, 2006, pp. 14-14.

[26] R. T. Ma, S. Lee, J. Lui, and D. K. Yau, "Incentive and service dif- 1472 ferentiation in P2P networks: A game theoretic approach," IEEE/ 1473 ACM Trans. Netw., vol. 14, no. 5, pp. 978-991, Oct. 2006.

[27] E. Palomar, A. Alcaide, A. Ribagorda, and Y. Zhang, "The peer's 1475 Dilemma: A general framework to examine cooperation in pure 1476 peer-to-peer systems," Comput. Netw., vol. 56, no. 17, pp. 3756- 1477 3766, 2012

[28] Y. Wang, A. Nakao, A. V. Vasilakos, and J. Ma, "P2P soft security: 147 On evolutionary dynamics of P2P incentive mechanism," Comput. 1480 Commun., vol. 34, no. 3, pp. 241-249, 2011

[29] B. Q. Zhao J. Lui, and D-M. Chiu, "A mathematical framework for 1482 analyzing adaptive incentive protocols in P2P networks," IEEE 1483 Trans. Netw., vol. 20, no. 2, pp. 367-380, Apr. 2012.

[30] R. Mahajan, M. Rodrig, D. Wetherall, and J. Zahorjan, 1485 "Experiences applying game theory to system design," in Proc. 1486 ACM SIGCOMM Conf. Appl. Technol. Archit. Protocols Comput. 1487 Commun., 2004, pp. 183-190.

[31] R. B. Myerson, Game Theory. Cambridge, MA, USA: Harvard 148 Univ. Press, 2013

[32] J. Weibull, Evolutionary Game Theory. Cambridge, MA, USA: MIT Press, 1997. [Online]. Available: https://github.com/glenacota/racoon 1494

[34] A. Yumerefendi and J. S. Chase, "The role of accountability in 1495 dependable distributed systems," in Proc. 1st Conf. Hot Topics Syst. 1496 Dependability, 2005, pp. 3-3.

[35] M. Belenkiy, et al., "Making P2P accountable without losing 1498 privacy," in Proc. ACM Workshop Privacy Electron. Soc., 2007, 1499 pp. 31-40

[36] F. D. Garcia and J. H. Hoepman "Off-Line karma: A decentralized 1501 currency for peer-to-peer and grid applications," in Proc. 3rd Int. 1502 Conf. Appl. Cryptography Netw. Secur., 2005, pp. 364-377.

37] S. Marti and H. Garcia-Molina, "Taxonomy of trust: Categorizing 1504 P2P reputation systems," Comput. Netw., vol. 50, no. 4, pp. 472- 1505 $484,2006$.

[38] K. Hoffman, D. Zage, and C. Nita-Rotaru "A survey of attack and 1507 defense techniques for reputation systems," ACM Comput. Sur- 1508 veys, vol. 42, no. 1, 2009, Art. no. 1 .

[39] S. Naicken, B. Livingston, A. Basu, S. Rodhetbhai, I. Wakeman, 1510 and D. Chalmers, "The state of peer-to-peer simulators and simu- 1511 lations," ACM SIGCOMM Comput. Commun. Rev., vol. 37, pp. 95- 1512 98, 2007.

[40] C-E. Killian, J. W. Anderson, R. Braud, R. Jhala, and A. M. Vahdat, 1514 "Mace: Language support for building distributed systems," in 1515 Proc. 28th ACM SIGPLAN Conf. Program. Language Des. Implemen- 1516 tation, 2007, pp. 179-188.

[41] L. Leonini, E. Rivière, and P. Felber, "SPLAY: Distributed systems 1518 evaluation made simple (or how to turn ideas into live systems in 1519 a breeze)," in Proc. 6th USENIX Symp. Netw. Syst. Des. Implementa- 1520 tion, 2009, pp. 185-198.

[42] G. Lena Cota, et al., "Analysing selfishness flooding with SEINE," 1522 to appear in Proc. 47th IEEE/IFIP Int. Conf. Dependable Syst. Net., 1523 2017.

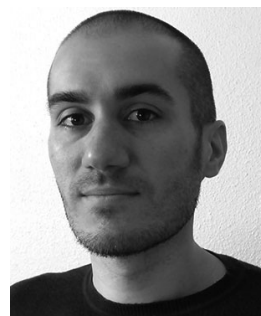

Guido Lena Cota received the $\mathrm{PhD}$ degree in 1525 computer science co-supervised by Università 1526 degli Studi di Milano (Italy) and INSA Lyon 1527 (France). His research interests include the areas 1528 of dependable and selfishness-resilient coopera- 1529 tive distributed systems. He is member of the 1530 IRIXYS Int. Research and Innovation Center. 


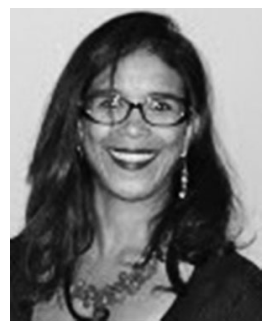

Sonia Ben Mokhtar received the PhD degree from the University Pierre et Marie Curie (Paris 6 ), in 2007, which she did under the supervision of Valrie Issarny and Nikolaos Georgantas in the INRIA ARLES project-team. She is a CNRS researcher at the LIRIS Lab, DRIM Group, since October 2009. Before that, she was a research associate with the University College London (UCL) for two years, working with Licia Capra. Her research interests include reliable distributed systems and middleware for mobile environments. She is member of the IRIXYS Int Research and Innovation Center.

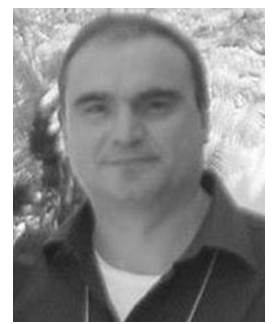

Gabriele Gianini received the $\mathrm{PhD}$ degree in physics from the Università degli Studi di Pavia. $\mathrm{He}$ is an assistant professor in the Department of Computer Science, Università degli Studi di Milano. His research interests include game theoretic applications to networking and security, quantitative modeling of processes, and statistical and soft computing techniques. $\mathrm{He}$ is a coordinator of the IRIXYS Int. Research and Innovation Center.

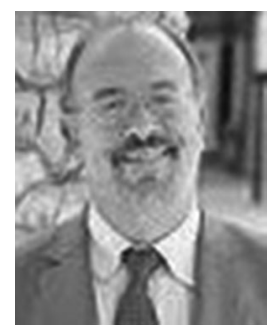

Ernesto Damiani received the $\mathrm{PhD}$ degree in computer science from the Università degli Studi di Milano, and the doctorate honoris causa from INSA Lyon. He is a full professor with the Università degli Studi di Milano and the director of the Information Security Research Center, Khalifa University, Abu Dhabi. His research interests include cloud assurance, Web services and business process security, and big data processing. $\mathrm{He}$ is a leading scientist of the IRIXYS Int. Research and Innovation Center.

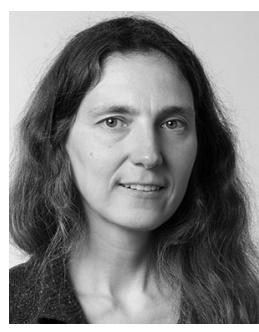

Julia Lawall received the $\mathrm{PhD}$ degree in com- 1567 puter science from Indiana University and was 1568 previously on the faculty of the University of 1569 Copenhagen. She is a senior researcher with 1570 Inria. Her research interests include program 1571 analysis and language design, applied to systems 1572 code.

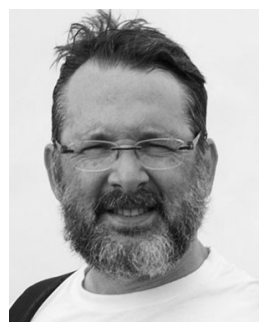

Gilles Muller received the PhD degree from the 1574 University of Rennes I, in 1988 and the habilita- 1575 tion a Diriger des Recherches degree from the 1576 University of Rennes I, in 1997. He is currently a 1577 senior research scientist with Inria Paris and the 1578 head of the Whisper Group. His research inter- 1579 ests include the development of methodologies 1580 based on domain-specific languages for the 1581 structuring of infrastructure software.

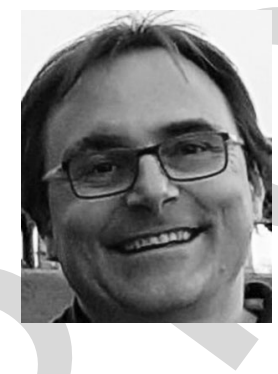

Lionel Brunie is a professor in the National Insti- 1583 tute of Applied Sciences of Lyon, France. His 1584 main research interests include data manage- 1585 ment and resilience in distributed systems, and 1586 information privacy and security. He is a leading 1587 scientist of the IRIXYS Int. Research and Innova- 1588 tion Center.

$\triangleright$ For more information on this or any other computing topic, 1590 please visit our Digital Library at www.computer.org/publications/dlib. 1591 